Article

\title{
Representation and Preservation of Heritage Crafts
}

\author{
Xenophon Zabulis ${ }^{1, * \mathbb{D}}$, Carlo Meghini ${ }^{2}$, Nikolaos Partarakis ${ }^{1}$, Cynthia Beisswenger ${ }^{3}$, \\ Arnaud Dubois ${ }^{4}$, Maria Fasoula ${ }^{5}$, Vito Nitti ${ }^{6}$, Stavroula Ntoa ${ }^{1}$, Ilia Adami ${ }^{1}$, \\ Antonios Chatziantoniou ${ }^{1}$, Valentina Bartalesi ${ }^{2}$, Daniele Metilli ${ }^{2}$, Nikolaos Stivaktakis ${ }^{1}$, \\ Nikolaos Patsiouras ${ }^{1}$, Paraskevi Doulgeraki ${ }^{1}$, Effie Karuzaki ${ }^{1}$, Evropi Stefanidi 1,7, \\ Ammar Qammaz 1,7, Danae Kaplanidi ${ }^{5}$, Ilka Neumann-Janßen ${ }^{3}$, Ulrike Denter ${ }^{3}$, \\ Hansgeorg Hauser ${ }^{3}$, Argyro Petraki ${ }^{1}$, Ioannis Stivaktakis ${ }^{1,7}$, Eleni Mantinaki 1,7, \\ Anastasia Rigaki ${ }^{1,7}$ and George Galanakis ${ }^{1,7}$ \\ 1 Institute of Computer Science, Foundation for Research and Technology (ICS-FORTH), N. Plastira 100, \\ Vassilika Vouton, GR-700 13 Heraklion, Crete, Greece; partarak@ics.forth.gr (Nikolaos Partarakis); \\ stant@ics.forth.gr (S.N.); iadami@ics.forth.gr (I.A.); hatjiant@ics.forth.gr (A.C.); nstivaktak@ics.forth.gr (N.S.); \\ patsiouras@ics.forth.gr (Nikolaos Patsiouras); vdoulger@ics.forth.gr (P.D.); karuzaki@ics.forth.gr (E.K.); \\ evropi@ics.forth.gr (E.S.); ammarkov@ics.forth.gr (A.Q.); argpet@ics.forth.gr (A.P.); \\ johnstiv@ics.forth.gr (I.S.); csd3566@csd.uoc.gr (E.M.); rigaki@ics.forth.gr (A.R.); ggalan@ics.forth.gr (G.G.) \\ 2 Istituto di Scienza e Tecnologie della Informazione (ISTI), Consiglio Nazionale delle Ricerche (CNR), Area \\ della Ricerca CNR, via G. Moruzzi 1, 56124 Pisa, Italy; carlo.meghini@isti.cnr.it (C.M.); \\ valentina.bartalesi@isti.cnr.it (V.B.); metilli@isti.cnr.it (D.M.) \\ 3 Förderverein Haus der Seidenkultur (HdS), Paramentenweberei Hubert Gotzes e.V., Luisenstraße 15, \\ 47799 Krefeld, Germany; cynthia.beisswenger@seidenkultur.de (C.B.); \\ ilka.neumann88@googlemail.com (I.N.-J.); museum@seidenkultur.de (U.D.); hhf38kr@gmail.com (H.H.) \\ 4 Histoire des technosciences en société, Conservatoire national des arts et métiers (HT2S-CNAM), Case \\ 1LAB10, 2 rue Conté, 75003 Paris, France; arnaud64.dubois@gmail.com \\ 5 Piraeus Bank Group Cultural Foundation, 6 Ang. Gerontas St., 10558 Athens, Greece; \\ FasoulaM@piraeusbank.gr (M.F.); danae.kaplanidi@gmail.com (D.K.) \\ 6 Imaginary, Piazza Caiazzo, 320124 Milano (MI), Italy; vito.nitti@i-maginary.it \\ 7 Computer Science Department, University of Crete, Voutes Campus, 70013 Heraklion, Crete, Greece \\ * Correspondence: zabulis@ics.forth.gr
}

Received: 17 January 2020; Accepted: 9 February 2020; Published: 15 February 2020

\begin{abstract}
This work regards the digital representation of tangible and intangible dimensions of heritage crafts, towards craft preservation. Based on state-of-the-art digital documentation, knowledge representation and narrative creation approach are presented. Craft presentation methods that use the represented content to provide accurate, intuitive, engaging, and educational ways for HC presentation and appreciation are proposed. The proposed methods aim to contribute to HC preservation, by adding value to the cultural visit, before, and after it.
\end{abstract}

Keywords: cultural tourism; arts and crafts; intangible heritage

\section{Introduction}

Heritage Crafts (HCs), or crafts that are transmitted as Cultural Heritage $(\mathrm{CH})$, exhibit tangible and intangible dimensions. Tangible dimensions regard craft articles and products, materials and tools, as well as natural resources, built workshops, and workplaces. The tangible, or material, aspect of HCs is evident in their practice, where materials are transformed with the use of tools, but also, skill and knowledge. From this perspective, "crafts are probably the most tangible of intangible heritage" [1]. Intangible dimensions regard technical knowledge, as well as the socio-historic content of 
the communities and regions in which they are, or were, practiced. HCs are part of the history and economic life of the regions and communities in which they flourish.

Comparatively to other forms of Intangible Cultural Heritage (ICH), $\mathrm{HCs}$, have received smaller attention by digitisation projects. Several HCs are threatened with extinction, due to the declining numbers of practitioners and apprentices. Despite the cultural significance of HCs, pertinent safeguarding and preservation efforts are scattered geographically and thematically. The ICH, due to traditional craftsmanship is a component of regional culture and identity, which directly relates to a natural heritage of a place and other forms of heritage that region hosts. In contrast to material heritage that can be sensed, intangible heritage is experienced. Digital media technologies facilitate the authoring of narratives and storytelling that emotionally engage the public. This is relevant in creating experiences that help us understand, value, and enjoy cultural heritage.

Cultural tourism refers to travel that enables visitors to visit heritage sites and activities that provide access to the $\mathrm{CH}$ of a country or region. The reason a region develops tourism is not anymore solely related to the particularities of the natural environment and built sites, but also to its their cultural identity, tourism infrastructure, and services. In particular, cultural tourism related to traditional crafts exhibits the following potentials:

1. Craft articles have multiple affordances as traded products. Typically, they are utilitarian objects. As heritage articles, they carry tradition and aesthetics. As souvenirs, we associate them with our own memories of presence at a place or event. Last, self-made craft articles in participatory engagement provide experiences and embodied knowledge.

2. Craft workshops and pertinent thematic tourism activities exhibit the potential of participatory experiences and access to local culture. Thematic tourism and workshops can extend visitor stay on location or enhance the options of day-trips near central sites.

3. Thematic tourism alleviates visitor load from mass tourism destinations. Crafts flourish in multiple, distributed, rural areas. Unlike monuments of material heritage, intangible forms of heritage, have a wider spatial distribution as to the locations that can be practiced.

The rationale of the proposed approach is that attractive, participative, educational, and experiential and tourism products are expected to motivate the preservation of HCs. In addition, the geographical context is required to be considered to provide means of collaboration with existing neighbouring cultural sites, geo-parks, and workshops.

Cultural and thematic tourism can be a motive for the preservation of HCs, given that stakeholders benefit from it. To achieve this on a distributed, per community basis, documentation and preservation of HCs are simplified by data collection tools and an approach to their semantic organisation and presentation. The proposed approach lowers the technical barriers to capacity building for $\mathrm{HC}$ communities.

In this work, we focus on representing tangible and intangible content, due to HCs, for uses that enhance the value of a cultural visit. This is strategized by providing educational content and engaging experience for uses prior, during, and after a visit. These aim to facilitate engagement with regional culture, using modern, intuitive, and attractive media that impact a wide range of audiences, including younger ages. The goal is to support the link between craft practice and new interest participatory tourism services that provide such experiences, through day visits or workshops. For this reason, this work proposes (a) a set of digitisation and analysis tools that facilitate the curation of content for educational visits and workshops and (b) systematic knowledge representation methods and presentation modalities, tailored for content relevant to HCs.

\section{Relevant Work}

\subsection{Creative and Cultural Industries and Their Contribution to the Economy}

The economic potential of the creative and cultural industries is underscored in a number of studies. The EC [2], revealed that the creative economy by 2003 created $2.6 \%$ of the EU GDP and 
employed around five million people. The report mentions that "culture drives economic and social development ", as also attested by the UN [3], and the EC [4]. In 2008, the creative economy created 8.5 million jobs across the EU (3.8\% of the total labour force) and 4.5\% of the European GDP [5]. In 2006, the global exports of crafts attained 186.5 billion USD [6]. According to figures from the International Tourism Organisation, cultural tourism accounts for $37 \%$ of tourist destinations and is growing by $15 \%$ annually. At European level, cultural tourism accounts for about $40 \%$ of all European tourism, and it is very interesting that it is not concentrated exclusively in major cities, but is spread throughout the European territory. Fifty percent of cultural activity in Europe is relevant to $\mathrm{CH}$, and cultural tourism is the component of tourism with the largest prospective growth [7].

The UNWTO [8] indicates that digitisation of $\mathrm{CH}$ supports preservation. The economic resource, due to heritage is a primary motivator and source of funding for the preservation of $\mathrm{CH}$. Cultural tourists are generally high-cost and highly-educated people who are interested in cultural activity at the destination [7]. Cultural tourism has moved away from the traditional interest in static museums and monuments and demands interactive experiences, including tangible and digital artefacts, as well as engagement with local communities. There is a strong need to satisfy demanding visitors, particularly young people who usually do not find interest unless engaged in activities or games [9]. In many cases, participants of thematic tourism have little knowledge of the place, but a strong intent to become aware and learn. Educational tools contribute to the preservation and long-term sustainability of the HC economy [2].

\subsection{Heritage Digitisation}

Approaches towards heritage digitisation presented initial focus on artefacts and monuments of material heritage. Digitisation guides and good practice guidelines $[10,11]$ are now the norm in photographic documentation [12]. A decade later, the proliferation of surface digitisation technologies streamlined the 3D digitisation of artefacts and monuments. Digital 3D models of artefacts have a wide range of uses, from conservation and preservation to reunification of dispersed heritage [13]. File management, digital preservation, publication, and IPR management guides can be found in [14]. A significant online repository of material heritage is Europeana [15]. In the domain of verbal and numerical documentation, as well as meta-data, the CIDOC-CRM [16], ISO 21127:2014, is the de facto standard in the representation of heritage documentation.

Digitisation of intangible heritage attracted attention more recently. Human motion is a key component of many forms of ICH, such as dances, crafts, and rituals. Motion recordings were used to document and capture performances in i-Treasures [17], MODUL DANCE [18], DANCE [19], European Theatre lab [20], and TERPSICHORE [21]. Digitisation of human motion is achieved by a number of modalities, which vary in terms of pervasiveness (having to wear an apparatus or requiring an installation) and robustness to occlusions if visual components are employed.

\subsection{Craft Digitisation and Representation}

Crafts bring together the intangible dimensions of dexterity, skill, tradition, and knowledge, with tangible elements such as tools, machines and materials to be transformed into craft articles.

In the humanities, literature exists on the documentation of specific craft instances through ethnography, e.g., [22,23]. A significant work towards generic approaches to craft representation identifies tangible and intangible dimensions [24]. From the intangible dimensions, some relate to the cultural, economic, or religious contexts that identify communities. Others relate to the technical context of a specific craft and the corresponding skills, knowledge (or "know-how"). In [25], social context, group membership, and social relations relevant to crafts are reviewed. Guidelines for preservation projects [26] stress the representation and consent of the pertinent community. In addition, intangible dimensions regard the resources of the environment that a craft flourishes, and as such, regard environmental, and climate context [27].

Mingei (http://www.mingei-project.eu/) is an Innovation Action in the Horizon 2020 Programme of the EC that proposes the digital and semantic representation of HCs and motivates their preservation, 
by supporting pertinent experiential and educational applications in the domain of cultural and thematic tourism.

In Mingei, a systematic approach to the digital representation of tangible and intangible dimensions that closely follows the work in [24], is proposed. In [28], the requirements towards such a representation are investigated, so that the represented content is sufficient to support authoring of accurate narratives, curation of educational material, and experiential presentations. In [29], an approach towards the specification, acquisition, and representation of pertinent data and knowledge for HC preservation is proposed. In [30], Motion Vocabularies are proposed to associate recordings of craft practice to craft actions and remap the recorded human motion to pieces of virtual machinery whose function is simulated. As a complement to Mingei, systems which leverage semantic information include automatic image annotation [31], and recommendations for users [32]. Such approaches could benefit from the knowledge database which Mingei aims to build.

In this work, we propose content representation and presentation methods that support craft-oriented thematic tourism, by providing digital content on craft articles and processes, as well as narratives on the cultural and regional context. This content is used as a basis for tourism, social, and educational activities with a positive impact on the sustainability of the transmitted heritage. A platform for digital applications is proposed, that can be used to author a comprehensive picture of the region of application. The project aims: (a) The unified promotion of intangible dimensions of craft products; (b) to enrich educational services of cultural tourism and ICH preservation; and (c) to enhance the visitor experience with participatory and cultural experiences. To the best of our knowledge, Mingei is the first approach for the systematic digital representation of HCs that encompasses tangible and intangible dimensions, in a semantic and machine-interpretable fashion. The proposed protocol, tools, and application guides have been designed with $\mathrm{CH}$ professionals, to simplify the representation process for stakeholders of the $\mathrm{CH}$ domain. As such, it contributes to the capacity building of pertinent $\mathrm{HC}$ communities.

\section{Method}

The proposed approach can be described as a series of iterative steps for representing a Craft Instance (CI), that is the expression of a craft at some geographical region and at a given time period. In the first steps, we select and acquire digital assets. These assets are digitisations of craft artefacts, documents, photographs, archives, practitioner recordings, and curated material.

The collection of data and process descriptions are based on ethnographic studies that conform to the individualities of each craft and its materials. The collaboration with craft practitioners and community members is deemed a prerequisite [26] for a meaningful representation of technical processes, as well as an emic understanding of cultural context. In this work, the ethnographic approaches utilised are obtained from [29].

In the next stage, information obtained from data analysis is curated and represented as basic knowledge. This knowledge is enhanced with semantic associations to create a thick representation, meaning a description of not only actions and events, but their context as well, so that they can be better understood. This representation is not a 'unique' representation of CI, but rather a representation possibility as authored by the curator of this knowledge.

In Section 4.1, the obtained CI representation is a knowledge base for retrieving content and curating narratives on the CI. In Section 4.2, narrative content is provided to presentation modalities, in the scope of tourism, education and preservation.

\subsection{Craft Representation}

We follow the approach in [29], to collect data and orient the selection of contextual knowledge. Our point of departure is a selection of topics that comprise a comprehensive picture of a $\mathrm{CI}$, its practice and products, as well as the geographical, historical, and societal context at the place of practice. The technical platform can host multiple such organisations, as individual descriptions, i.e., multiple 
techniques from individual practitioners for a given task. In this work, following the articulation of actions as described by the practitioner is proposed.

The proposed method assumes the semantic capability representation of knowledge. A technical platform for formal, machine-interpretable knowledge representation is required, which, in our case, is CIDOC-CRM [16].

The remainder of this subsection is organised as follows. First, a selection of cost-efficient digital recording modalities for the application domain of crafts is provided. Second, a representation approach is proposed, which associates recordings of craft practice with (i) a semantic articulation of actions of a craft process and (ii) the 3D motion of body members, objects or materials. Third, a way to represent social and historical knowledge relevant for a given $\mathrm{CI}$, the community of practitioners, and the place of its practice is presented.

The purpose of these modalities is a repository of digital assets and information, which is curated into a knowledge base that is articulated by basic knowledge elements. These are statements that explain the role and significance of the corresponding digital assets. These elements include any existing curated information or description about the asset. They are created by new data entry or linked to existing repositories.

\subsubsection{Digitisation}

Digitisation of objects and actions supports documentation and illustration of narratives, authoring of instructions, and training experiences. A breadth of physical objects is of relevance to a heritage craft, such as materials, tools, machines, protective garments, and working environment. In the following examples, a selection of material digitisation methods is proposed, orientated towards indoor workshops, rural environments, and suburban regions that are most often associated with $\mathrm{HC}$ and other, associated forms of ICH.

In Figure 1, a digitisation of rural structure the seasonal droving of livestock, for the hospitality of sheep and persons [33]. The structure is worth seeing in closer detail, as it is constructed by the technique of dry stone walling [34]. Combination of multiple scans from a range of modalities, (aerial and terrestrial photogrammetry, laser scanning) are employed to produce a realistic indoors and outdoors 3D reconstruction, which can be used to provide terrain overview and functional explanation of the settlement components from its inside. In a smaller scale, the digitisation of tools and machinery using such modalities is demonstrated in Figure 2. More specifically, for photogrammetry-based reconstruction a GoPro ${ }^{\circledR}$ Hero6 camera was used; also mounted on a DJI ${ }^{\circledR}$ Phantom 4 Pro V2.0 drone, in the case of aerial shots. Laser scans were obtained by either FARO ${ }^{\circledR}$ Focus M70 (static), or FARO ${ }^{\circledR}$ Freestyle 3D X (handheld) laser scanners. All equipment is owned by ICS-FORTH.
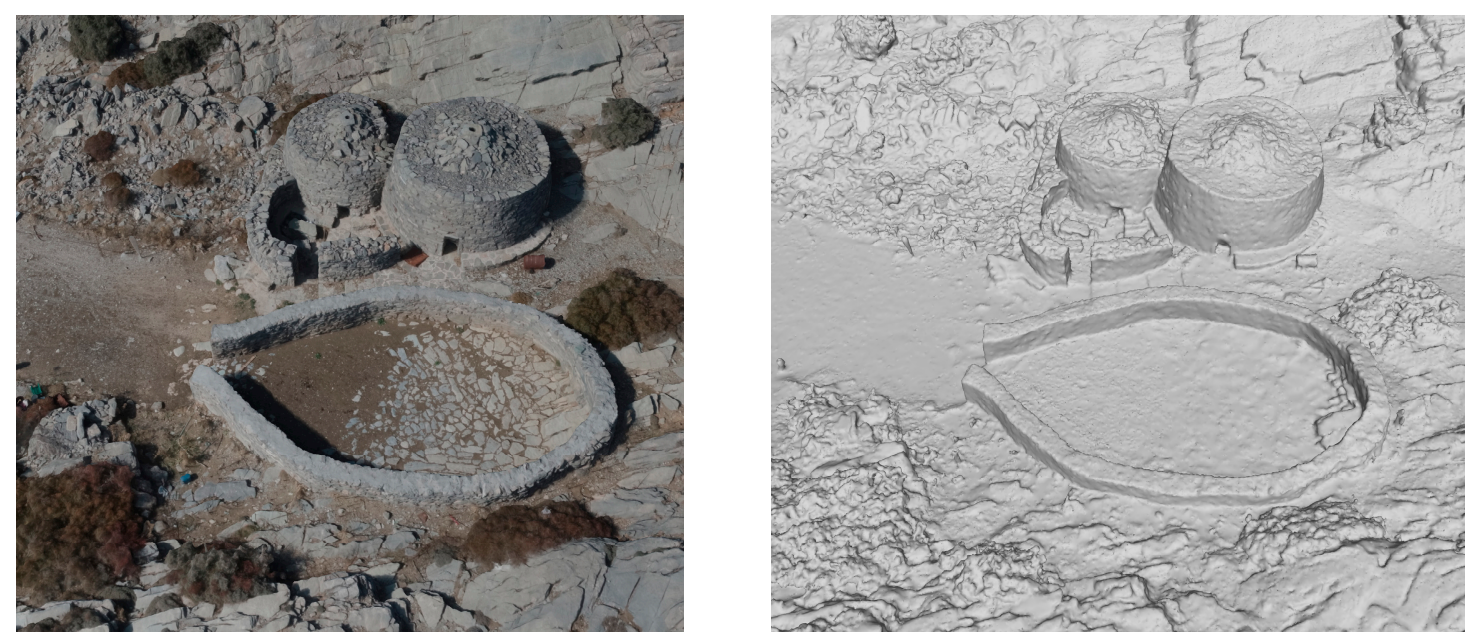

Figure 1. Cont. 

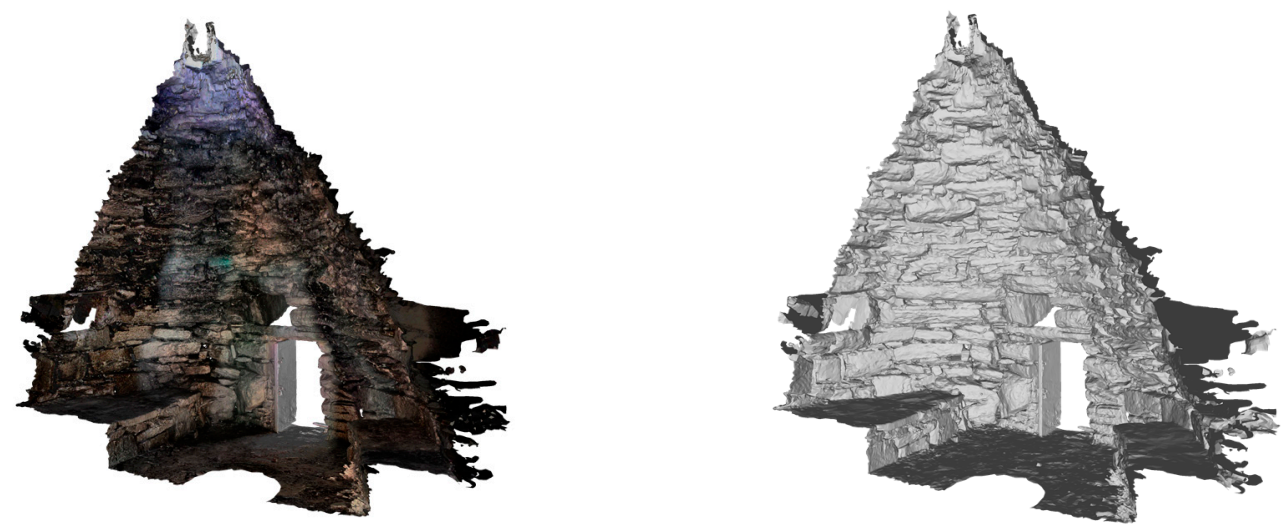

Figure 1. Digitisation of rural structure. Outdoors aerial scan (Top) Indoors laser scan (Bottom).
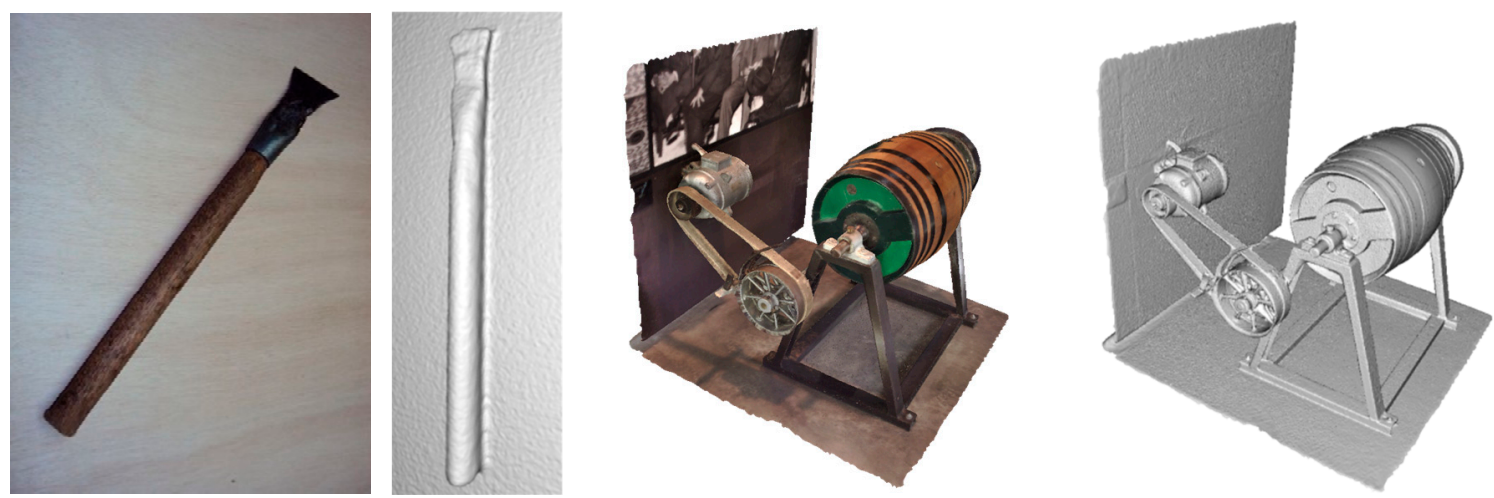

Figure 2. Digitisation of tools and machines.

In terms of digitisation resources, it is not necessary to treat every element with the utmost possible realism in terms of resolution, and thus, cost-efficient modalities, such as photogrammetry or handheld scanners suffice the goal. In some cases (see Section 3.2), it is more informative to show the function of a machine or the proper grip and manipulation of a tool, than vividly reconstructing its plain wooden handle in high resolution.

On the other hand, accurate digitisation is essential in the conservation of articles of material heritage, which serve as an exemplar of skill, technical knowledge, and aesthetics in its making. This is of particular relevance in the outdoors. This is the case in Figure 3, where a photogrammetric reconstruction of a sculpture of Tinian marble craftsmanship [35] is shown (The 3D reconstruction is provided in detail here: https://youtu.be/1hyTKpSIIUw). In this way, its condition can be compared to the condition which the artefact was photographed in the past.
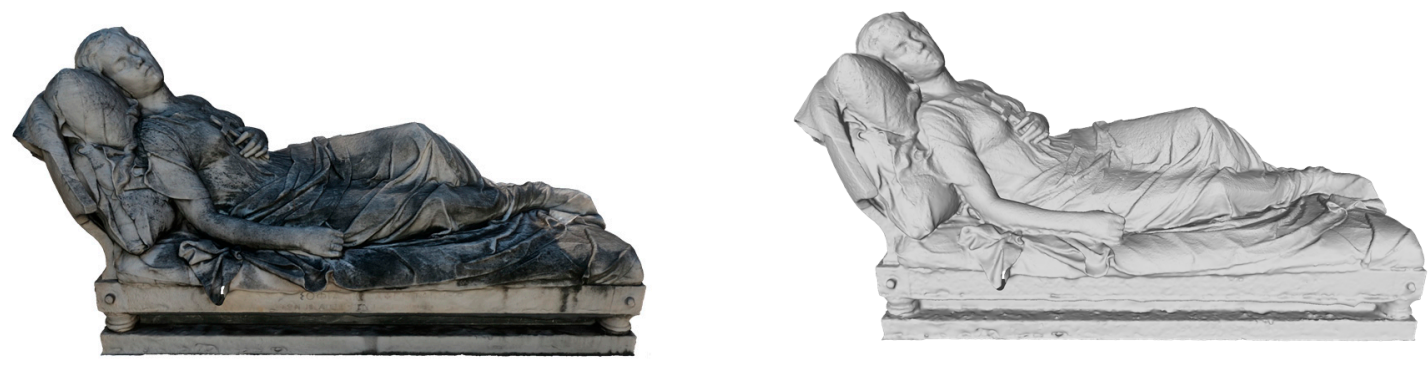

Figure 3. Cont. 

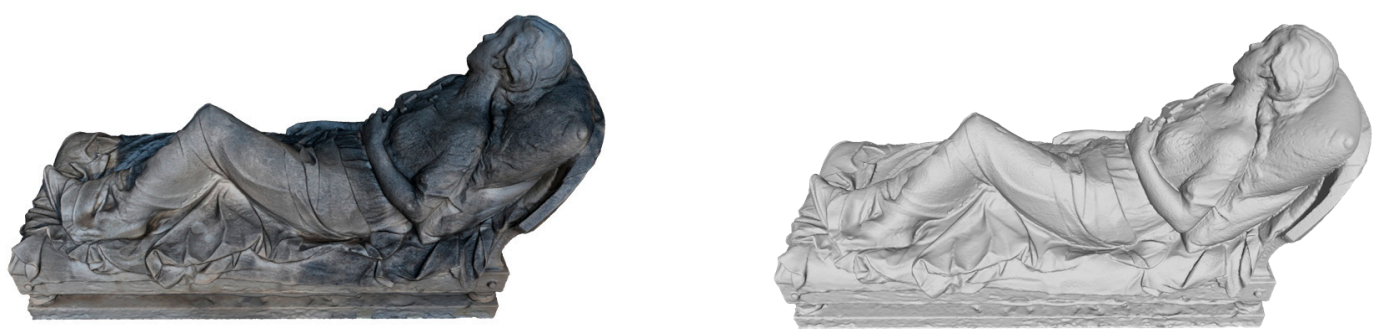

Figure 3. Photogrammetric reconstruction of a sculpture.

Relevant to craft representation is the digitisation of garments, either as products or as equipment. The top two rows of Figure 4, illustrate the rectification of a photogrammetric scan and its analysis in garment components using computer-aided 3D tools. The result can be later on "worn" on virtual characters or presented in detailed 3D illustrations. The last row, presents the very high resolution scanning of textiles in an experimental installation that was implemented. The installation is based on a Fused Filament Modelling printer combined with a macroscopic camera instead of the extruder and software that stitches neighbouring images generating image mosaics of very high resolution (https://openseadragon.github.io/openseadragonizer/?img=http\%3A\%2F\%2F139.91.186. 146\%2Fmedia\%2Fmulti-scale\%2Ftextile\%2F6.png\&encoded=true).
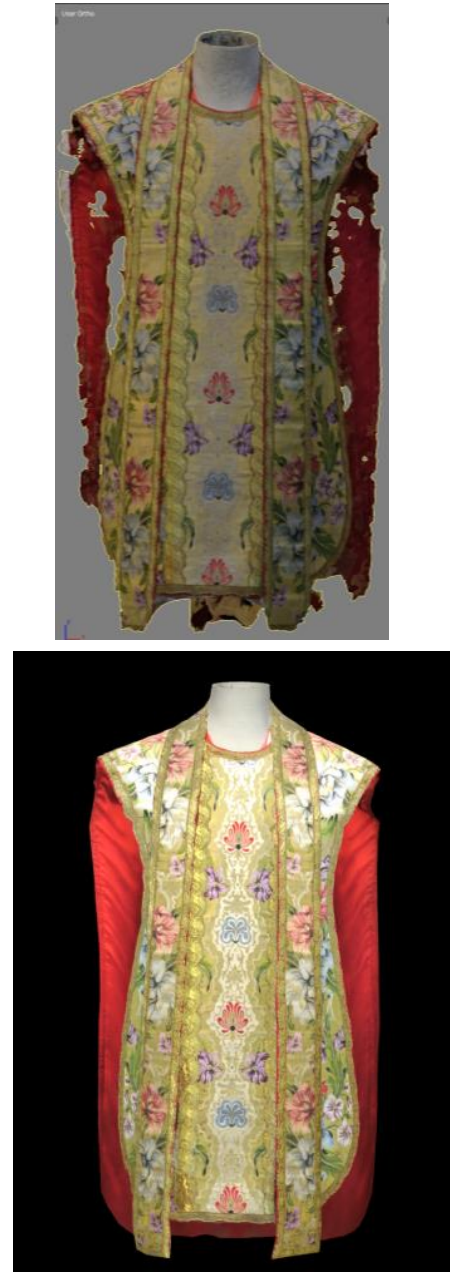
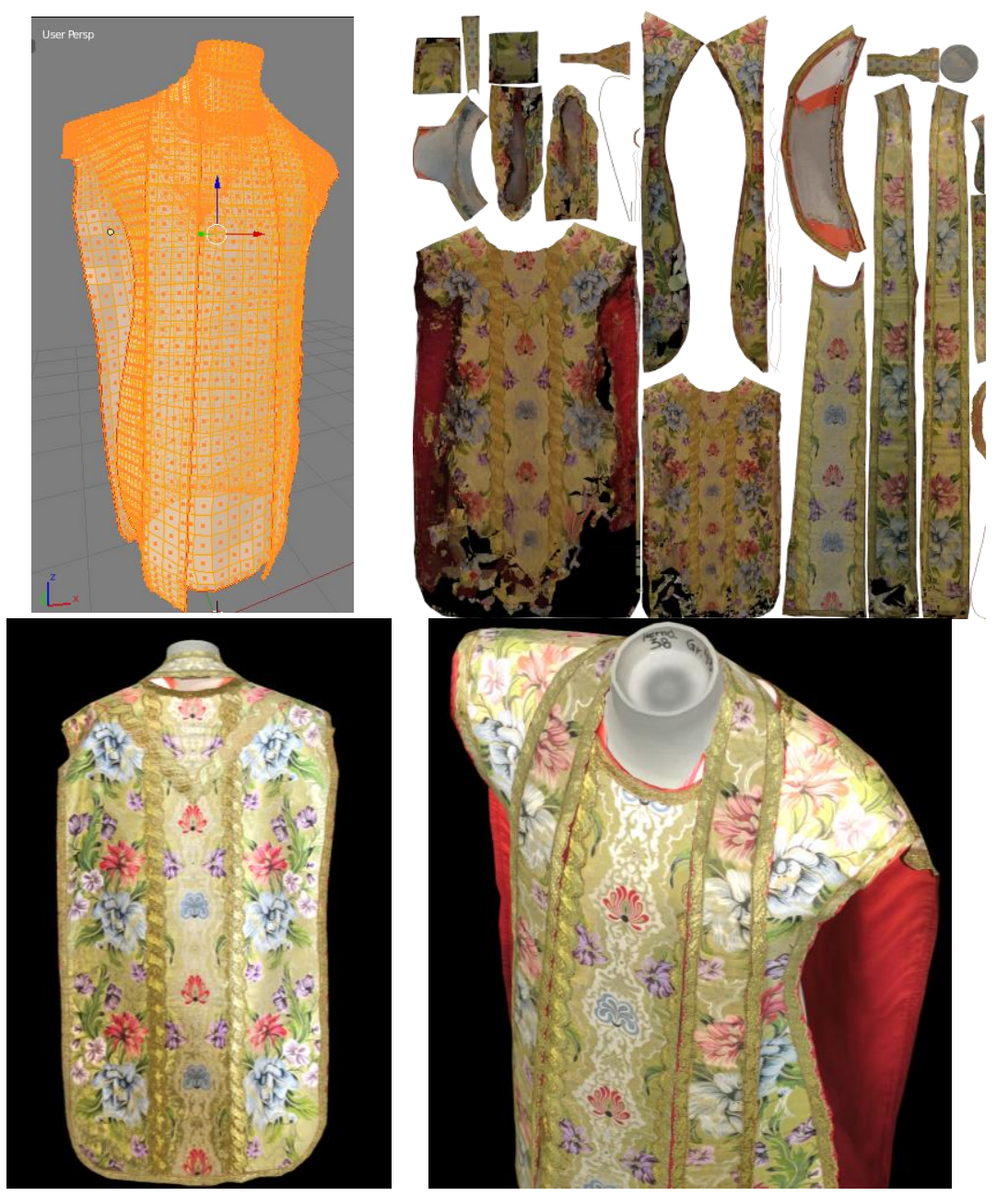

Figure 4. Cont. 

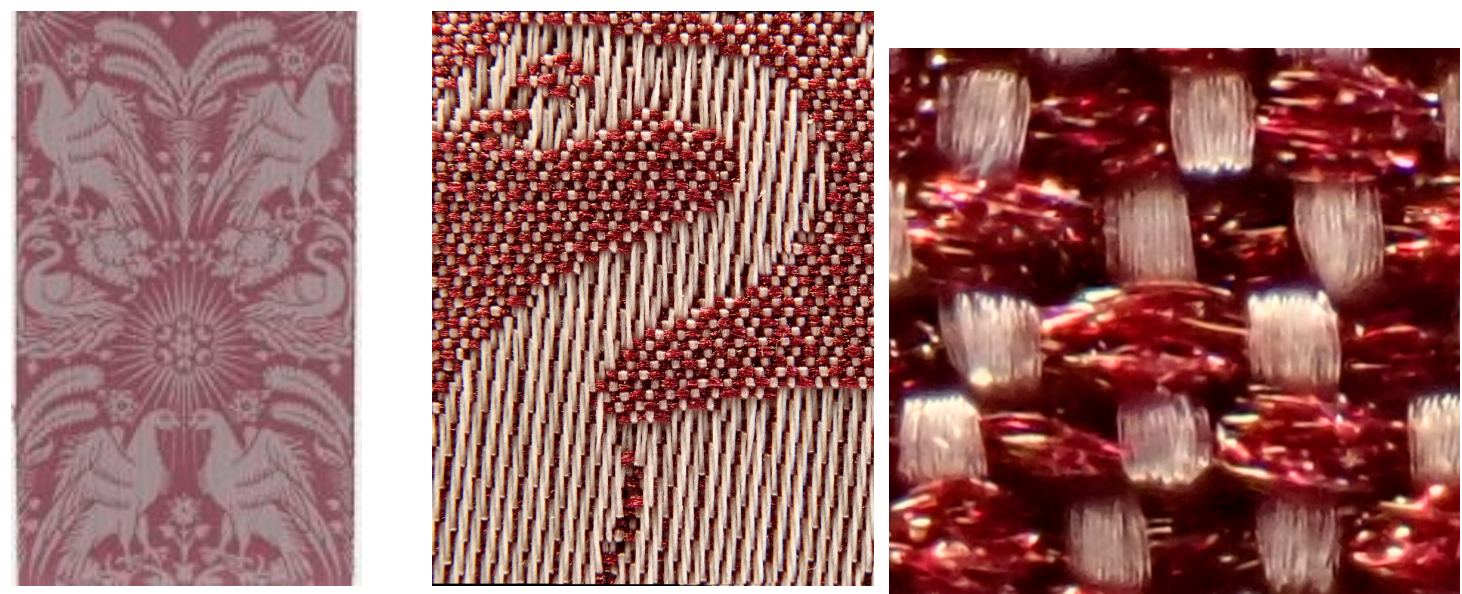

Figure 4. Garment and textile digitisation.

Craft practice is regarded as an activity that can be organised in actions and processes, where one or multiple practitioners use handicrafts and tools, to prepare or transform materials. Craft practice is organised in sequential and parallel steps, which we call activities. Our approach starts from the 3D digitisation of motion during craft practice.

Human motion recoding methods vary in terms of accuracy, pervasiveness, and robustness to visual occlusions. Recording is facilitated by inertial MoCap because visual methods are sensitive to occlusions, which are typical in the narrow and cluttered environments of workshops. Despite their sensitivity and post-processing requirements, we include visual methods in our digitisation modalities, due to the wide availability of CCD video. Visual methods are necessary when treating archive recordings (i.e., documentaries), when the wearable apparatus is restrictive for craft practice, and when inertial sensors are unusable, due to electromagnetic noise. Most importantly, visual methods enable the acquisition of data by non-experts.

In Figure 5, the top row, the wearable MoCap apparatus [36] used in the recordings is shown, and the 3D motion recordings are visualised. In Figure 5, the process and results of motion digitisation are visualised, for an inertial and a visual modality. On the bottom row, the threading of fragile, gold thread on a spool is shown. The body posture, thread grip, and periodic motion for homogeneous rolling of thread on a spool characterise this process. Motion is digitised in 3D by a visual approach [37], as footage comes for an archive documentary by Haus der Seidenkultur. The repetitive fine hand motion required to roll the thread on the spool is tracked using uniformly [38]. In the rightmost, this periodic gesture is summarised and explained by the MotiVo annotation editor (see Section 3.2).
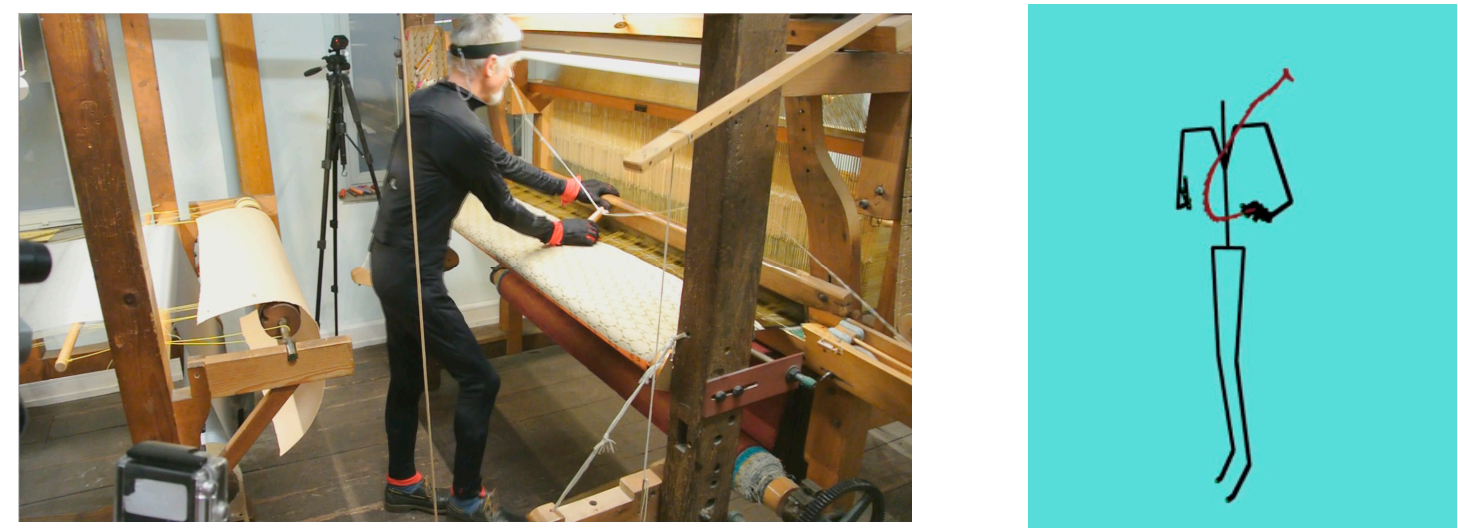

Figure 5. Cont. 

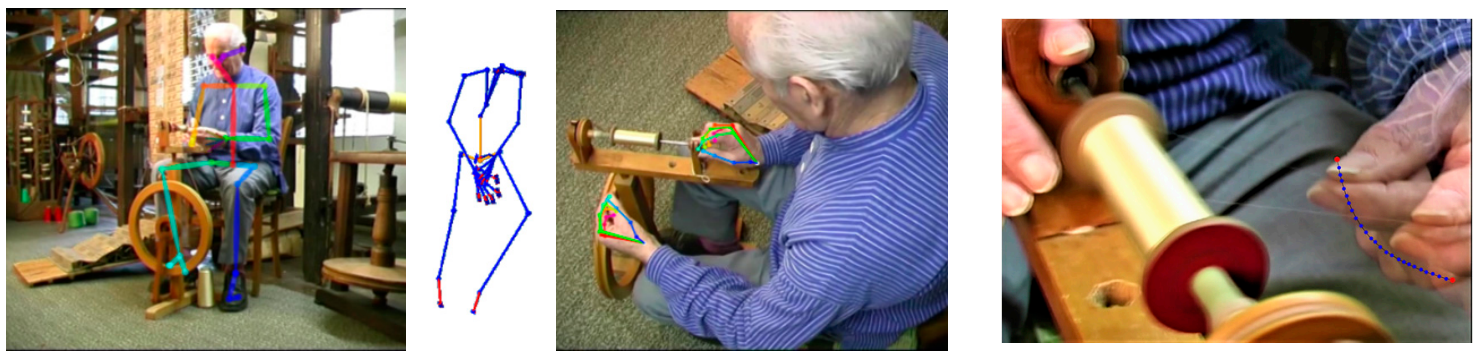

Figure 5. Visual human 3D motion estimation and 2D visualisation.

The platform facilitates the formation of knowledge elements for persons, events, objects, enterprises, and communities. Each element category is accompanied with a data collection form that allows users to add, modify and delete the respective elements. The users can view the elements of a category, add new ones, and delete the unwanted. Selecting an element allows to review and edit generic and category-specific information. Category-specific information refers to fields that are common within a specific category, such as the dimensions and the material for objects, birth and death information for persons and foundation date for enterprises. Generic information refers to common data across categories, such as the name, a short description, a display image, and media objects that are associated with that entity. Media Objects can be images, videos, motion captures, $3 \mathrm{D}$ reconstructions, 3D objects and motion vocabularies. They are authored independently and can be previewed to facilitate the association process. Entities and the data that accompany them are stored in a semantic knowledge base (see Figure 6).
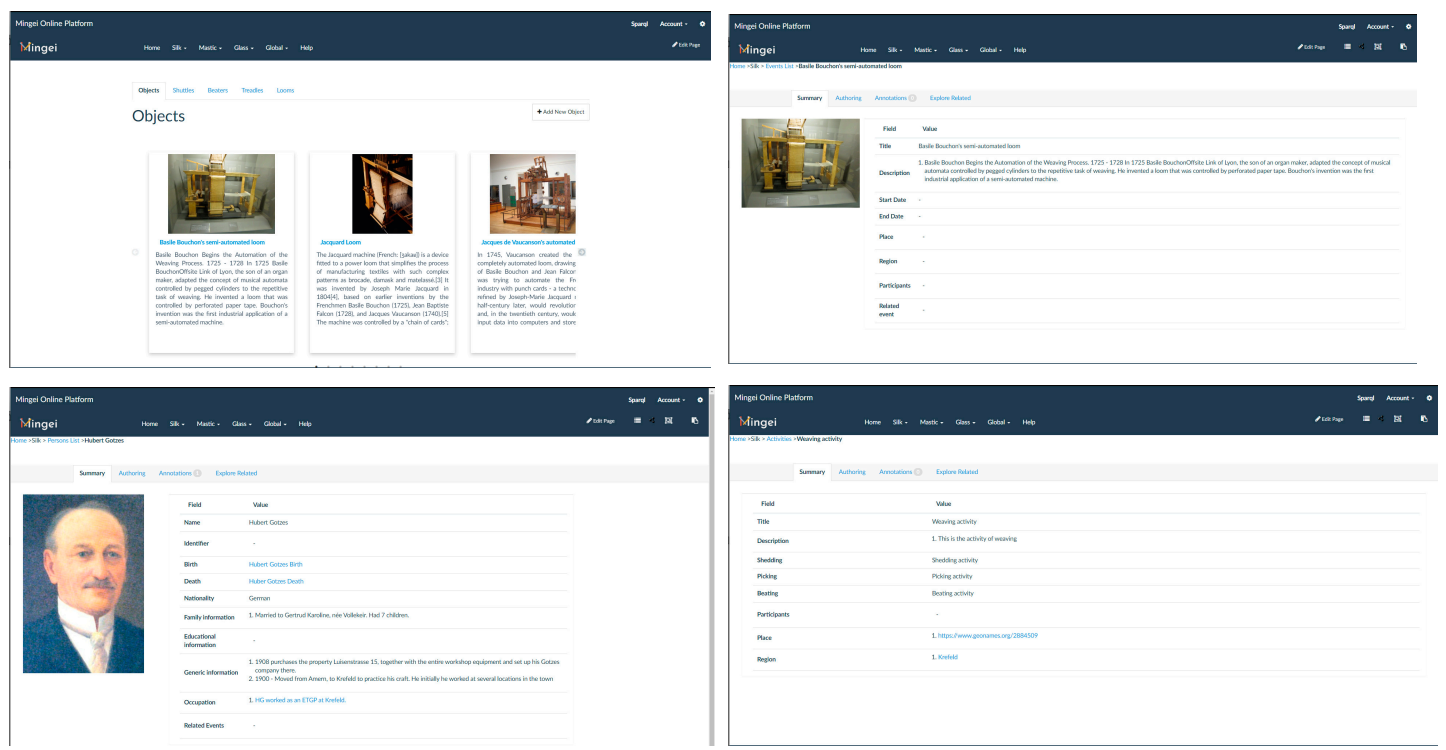

Figure 6. Knowledge element browsing and entry forms.

\subsubsection{Practice}

Craft activities are represented as the organisation of actions of craftspeople. Through ethnography, the relation of human, tool and material can be observed in detail to describe the process analytically. The analytical description leads to the representation of craft processes as process schemas. Process schemas are descriptions of the structures of activities that typically manifest themselves always following the same structure. Examples of such activities are wedding ceremonies, musical fugues, or soccer games. An occurrence of the schema is a set of (occurred) events that conforms to the schema. There is a vast amount of literature, and a number of standards in process schema representation, ranging from 
mathematical models, such as Petri Nets, to industrial standards, such as the Business Process Modelling Language. For the purposes of Mingei, we rely on UML activity diagrams, whose basic primitives have been, therefore, included in the Mingei ontology in the form of classes, properties and axioms on them.

The elements of process schemas are hypothetical actions, organised in processes. Inside a schema, actions are related to each other temporally, causally, and hierarchically. The implementation also contains links between the actions and conceptual objects. The digitisations of craft activities are recordings that contain these actions. Knowledge element digitisations are linked to conceptual actions as their instances. A tool facilitates the description of the craft process, analysing them in steps and defining the temporal or logical relationships between them. Each step and sub-step can be further analysed, thus, enabling the modelling of craft processes, whether linear and simple or complicated with a deep hierarchy of actions.

For each process the curator fills-in a title and a description. By default, the execution order for any process step is set to be "Any", not imposing any restrictions at first. All steps are listed in the order in which they were provided (see Figure 7). Once a step is listed, the curator defines its execution order, edit, or delete it. The tool provides support for additional material to describe each step in the form of textual guidelines, images, instructional videos, as well as the option to preview the graphical visualisation of the process as it has been defined in the form of UML diagrams.
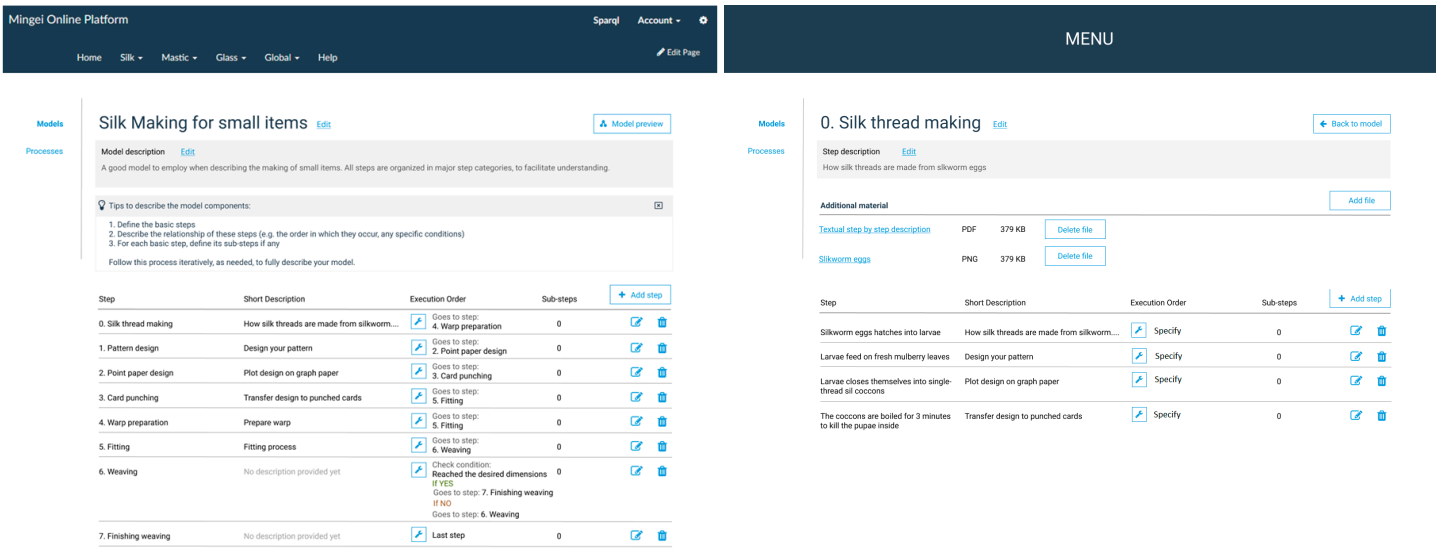

Figure 7. Example of a process (left) first level of steps (right) sub-steps of the first step.

The execution order options that are provided by the tool are explained in Table 1, the listing also the parameters that the user is asked to define for each one.

Table 1. Execution order options.

\begin{tabular}{clll}
\hline Order Option & \multicolumn{1}{c}{ Short Description } & & \multicolumn{1}{c}{ Parameters } \\
\hline Goes to step & Sequential ordering of steps & 1. & Next step \\
\hline $\begin{array}{c}\text { Goes to parallel } \\
\text { steps }\end{array}$ & $\begin{array}{l}\text { Connection of steps that run in } \\
\text { parallel }\end{array}$ & 1. & $\begin{array}{l}\text { Steps that will run in parallel. At least two steps } \\
\text { have to be defined. }\end{array}$ \\
\hline Waits for & $\begin{array}{l}\text { Connection of steps that run in } \\
\text { parallel and definition of the next } \\
\text { action once they are all completed }\end{array}$ & 2. & $\begin{array}{l}\text { Steps that have to be completed before taking } \\
\text { a next action. }\end{array}$ \\
$\begin{array}{clll}\text { Definition of the action that will follow, by selecting } \\
\text { an order option for the next action (goes to step, goes } \\
\text { to parallel steps, waits for, condition checking) }\end{array}$ \\
\hline $\begin{array}{c}\text { Condition } \\
\text { checking }\end{array}$ & $\begin{array}{l}\text { Denotes focal points in the process, } \\
\text { where the craftsman will check if a } \\
\text { condition is met before proceeding }\end{array}$ & $\begin{array}{l}\text { 1. } \\
\text { condion }\end{array}$ & $\begin{array}{l}\text { Condition that is examined } \\
\text { Execution order of steps if the condition is met } \\
\text { Execution order of steps if the condition is not met }\end{array}$ \\
\hline
\end{tabular}

Figure 8 illustrates the steps of defining the most complex option, that of condition checking. 

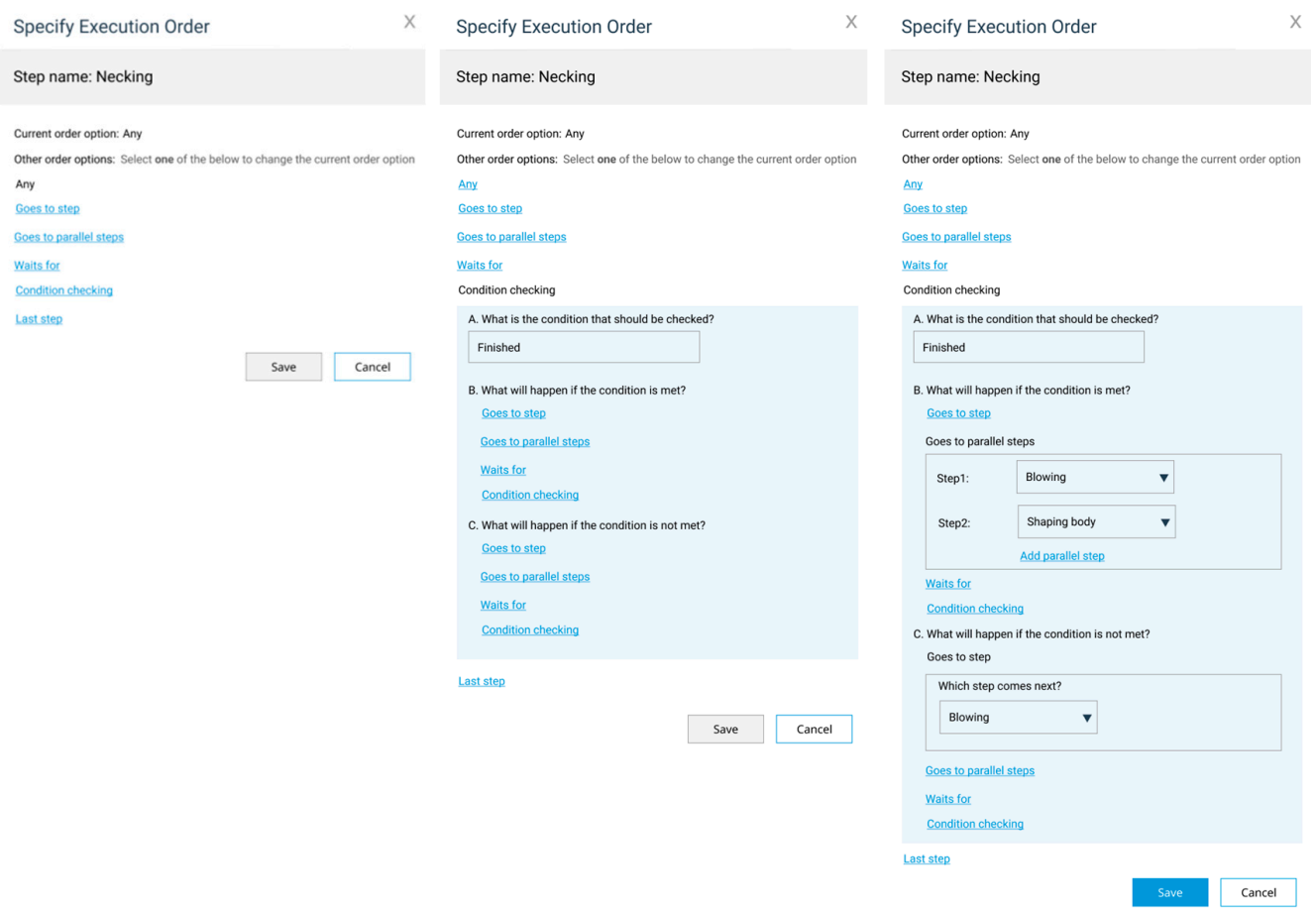

Figure 8. Definition steps of condition checking.

The representation implies delimitation of actions in high granularity. Recordings contain sequences of actions, some of which occur concurrently. An association between semantically represented action and fragments of digital assets is required. The representation of action may be relevant only to specific body members, e.g., the flying of a loom shuttle. Using computer-aided annotation on craft practice recordings, we create Motion Vocabulary Items associated with semantic descriptions of processes. This is facilitated by Animation Studio, an application implemented to visualise, edit, and annotate 3D animation files, obtained by motion capture or visual tracking. Pertinent annotation software exists in the linguistics domain [39], but not for 3D motion animations. Animation Studio facilitates isolation and annotation of animation segments and videos (see Figure 9).

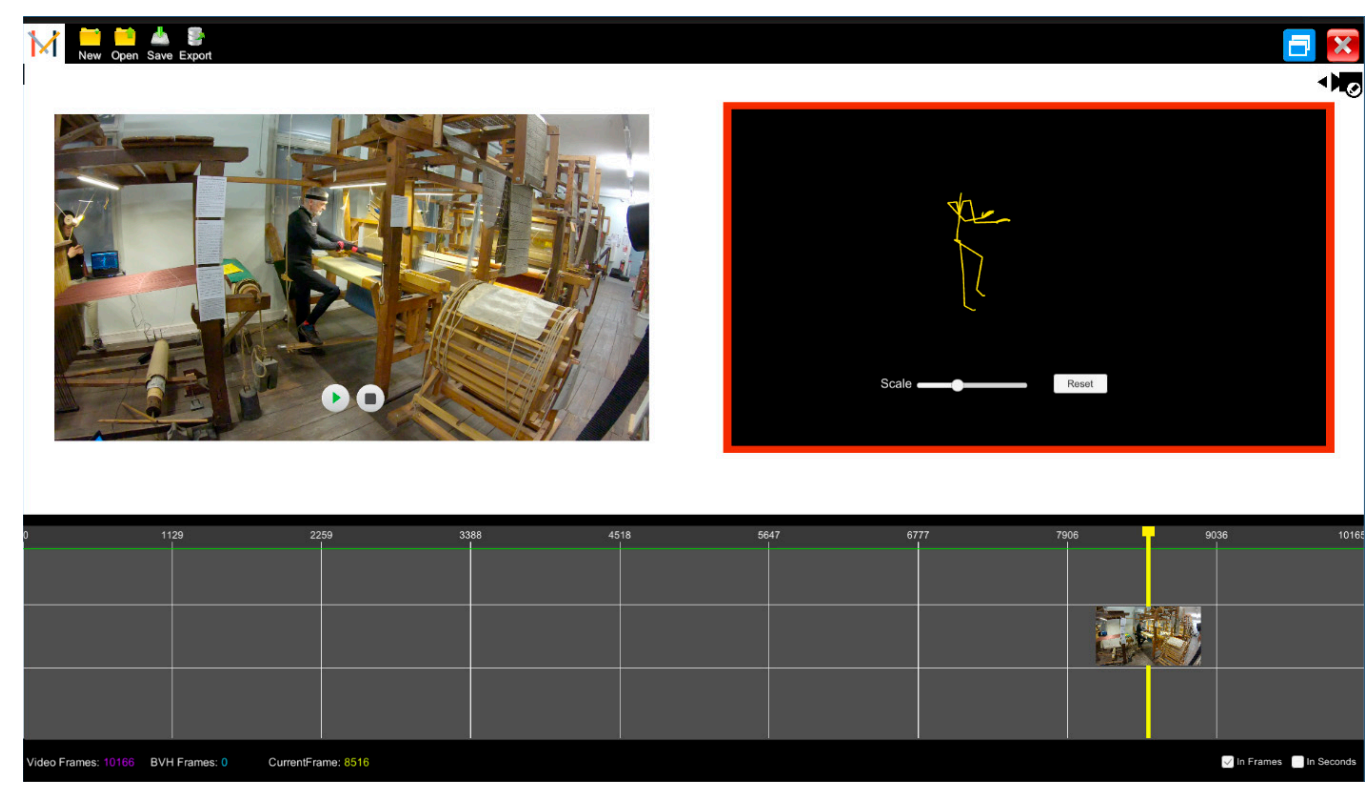

Figure 9. Animation Studio showing a visual stream, corresponding 3D motion data, and a timeline. 
Through the isolation of elementary physical actions from recordings of craft practice, we create Motion Vocabularies (MVs) that associate conceptual actions to recordings of these actions. In this case, the weaving process was decomposed into three actions, according to: (1) Shedding-warp threads are appropriately raised or lowered to form a shed; (2) picking - weft is passed across the shed using the shuttle; and (3) beating - weft is pushed against the fabric using the beater. The physical components of the loom are the shuttle, treadle and beater. The representation associates process steps with visual recordings and 3D motion of persons and objects, in a way that is intuitive to the practitioner and analytical for the representation of the process. This decomposition contains the interplay between human motion and interacting physical components (see Figure 10).

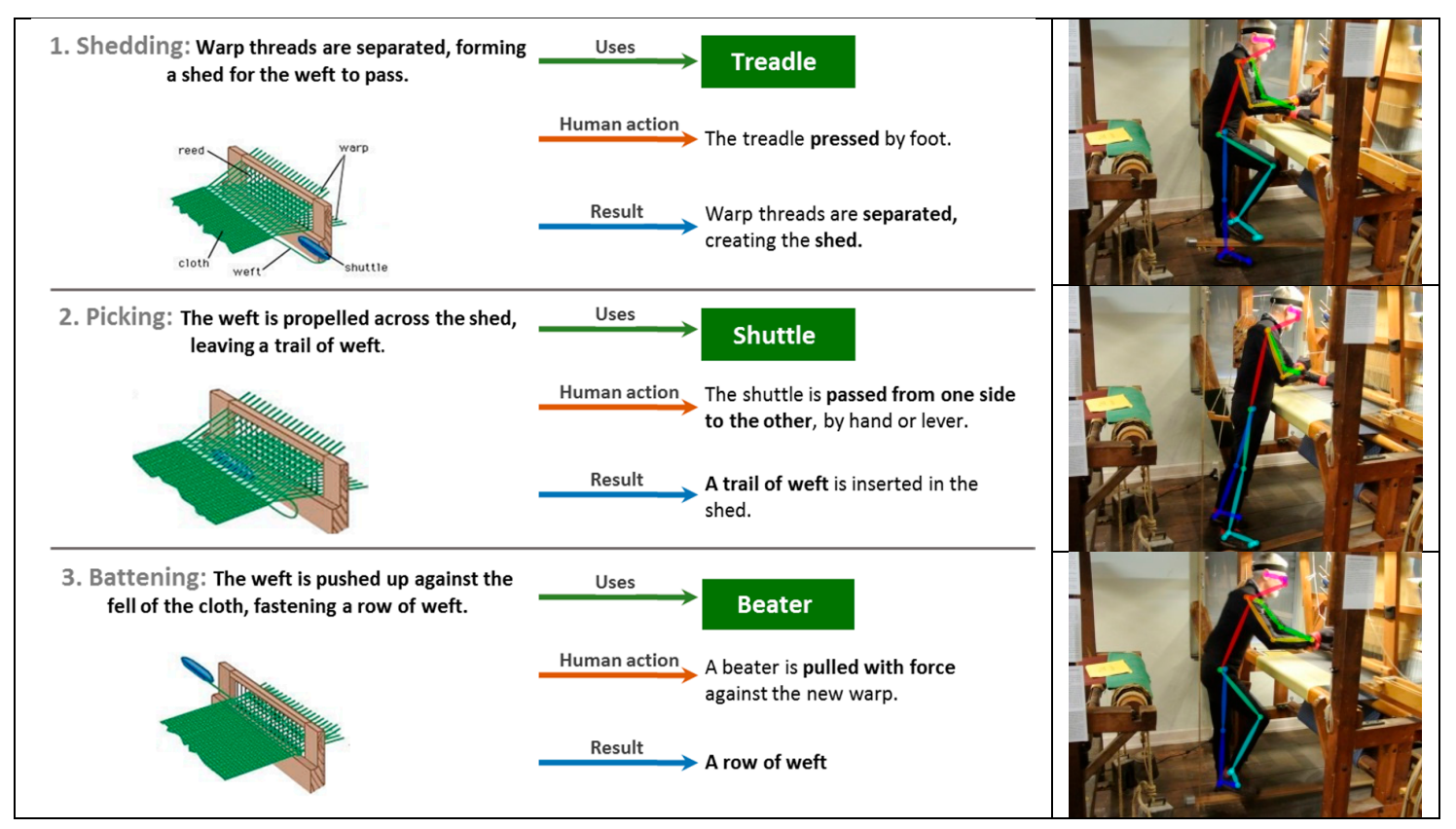

Figure 10. Process schema for weaving.

Similarly, manipulation of objects is associated with semantic processes, facilitated by the visual tracking of hands [38], and objects [40]. In Figure 11, the 3D model of the shuttle (left) is used to estimate its pose in 3D space and its interaction with the hands of the practitioner (right).
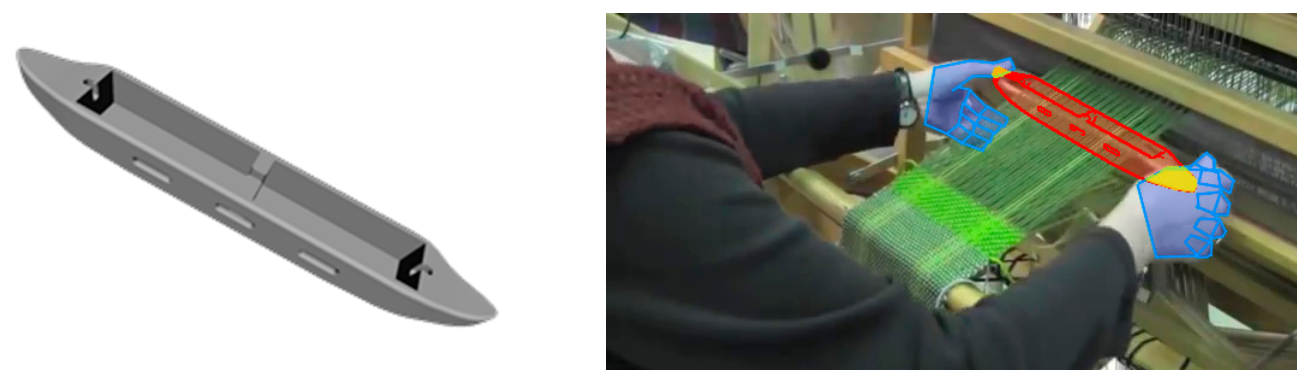

Figure 11. Hand and object pose estimation for visual recordings of craft practice.

To represent machine functionality, we decompose it in elementary components called Fundamental Machine Components (FMCs). These FMCs are 3D models enhanced with functionality that simulates their motion, implemented as described in [41]. As an example, we define the elements of the loom's physical interface as FMCs. Each one is comprised of a 3D model of the physical component and motion rules that represent its feasible motion during operation (see Figure 12). FMCs are paired 
with the body members used to operate them; e.g., treadle with foot. Mapping the motion recording to the FMC simulates the operation, by inference of the machine motion.
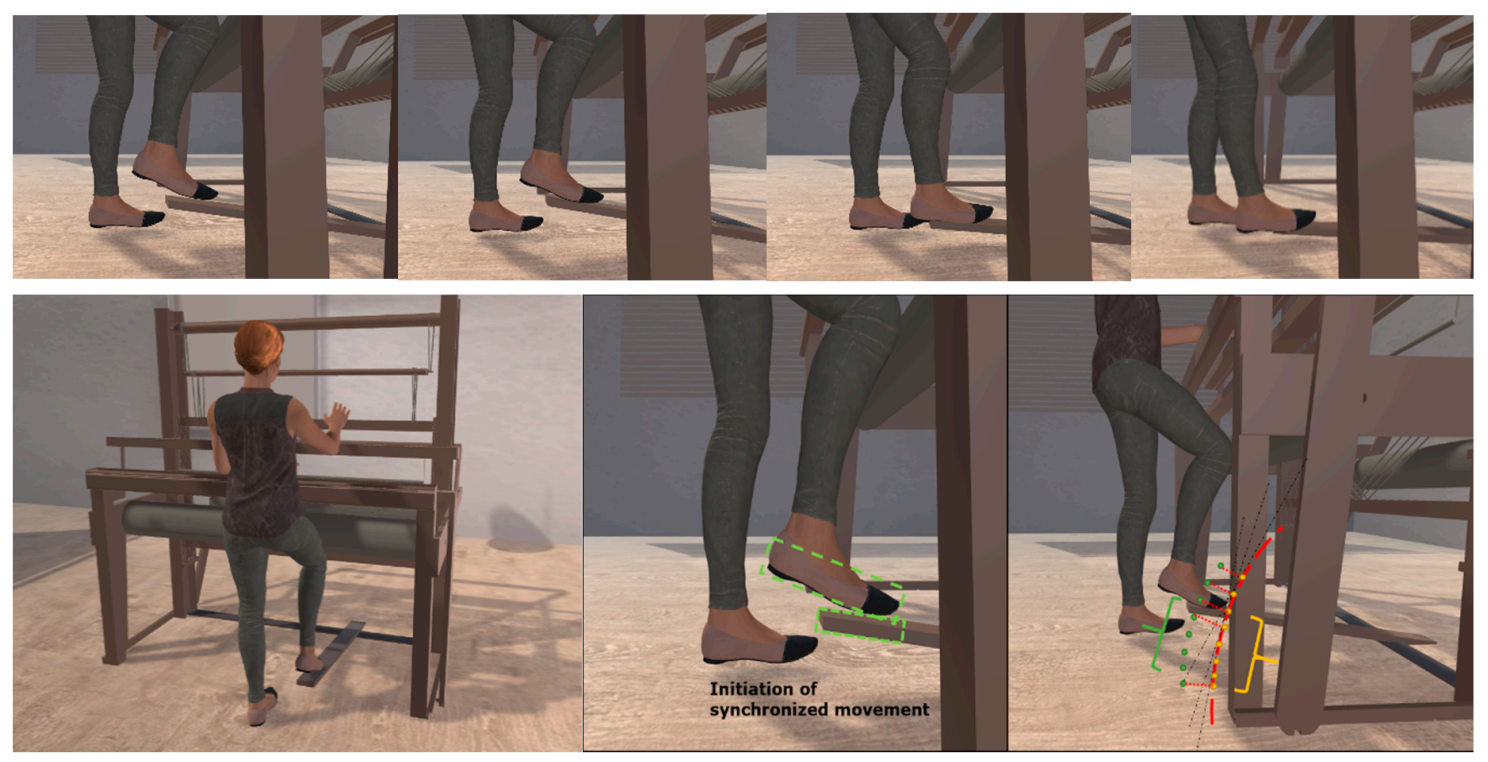

Figure 12. Visualisation of induced motion on a loom treadle.

\subsubsection{Context}

Bibliographic research and ethnography reveal the context in which a craft is or was practiced. Personal stories, social relations, and local history show the importance of a craft for a community and its region. Geographical context is relevant, as environmental aspects affect craft practice. The availability of materials depending on location is of relevance, such as when based on endemic flora or fauna. Temporal context is relevant to craft description, due to the change of season, technological progress, economic events, and climate change.

Context is represented through narratives, in the formal representation of the fabulae of narratives. In a narrative, the fabula is the piece of reality that contains the events narrated, in chronological order, as well as the facts that surround these events and that are immediately relevant to the events themselves, such as the people, objects, spatio-temporal regions involved in the events. In Mingei, each narrative comes equipped with a formal representation of the underlying fabula, expressed in terms of objects and inter-relationships. These objects and relationships form a semantic network, in which the fabula's events are explicitly represented as nodes and are linked to (a) the nodes that represent the people, objects, spatiotemporal regions involved in the events, and (b) the nodes that represent the media objects documenting the events. This rich, semantical representation of the fabula provides a contextualisation of the events composing the fabula. The contextualisation can be appreciated upon visualizing the narratives and can be used to build stories about the crafts and to link the crafts to other knowledge repositories via linked data mechanisms.

\subsection{Craft Presentation}

The Mingei project is investing a significant amount of resources to co-create storytelling scenarios for the crafts and the users addressed by the project. These include games and other activities that the users can enact in Virtual and Augmented reality scenarios, to appreciate the different phases, skills and techniques that compose the crafts. An online platform allows the visual representation of basic knowledge elements, such as events and places using interactive maps and timelines.

A classical storytelling component in narrative visualisation is the time-line visualisation, through which the user can appreciate the temporal placement of the events composing the narrative on 
an absolute scale. The site Mingei Online Platform provides automatic timeline visualisation of contextual events (Figure 13, left).

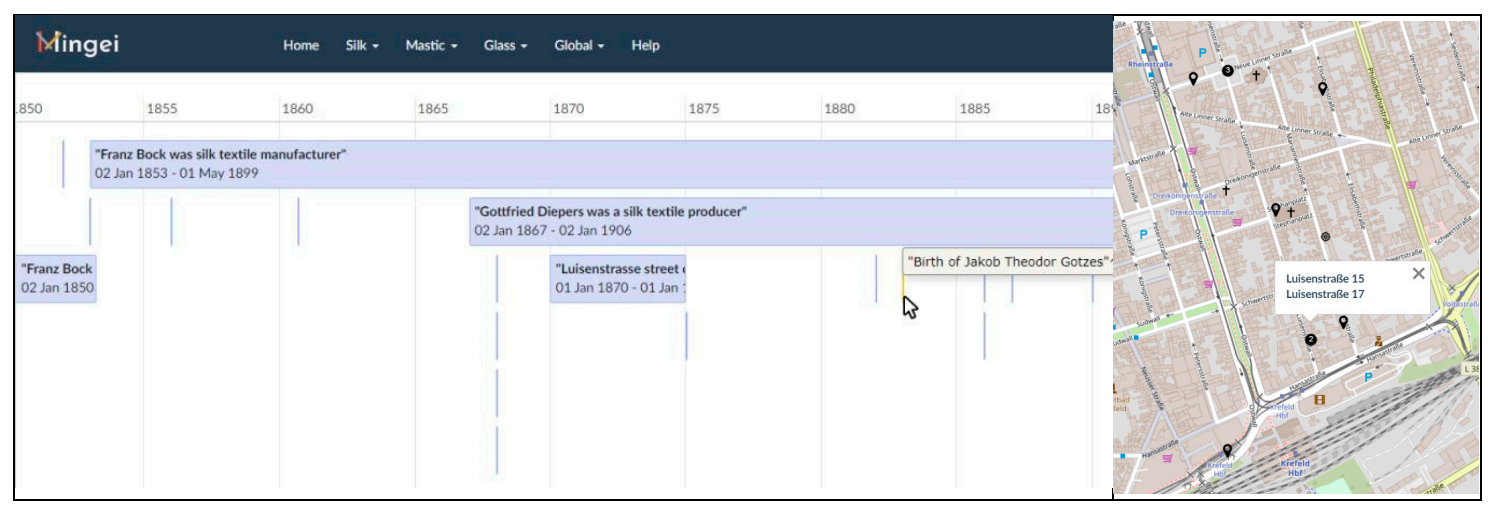

Figure 13. Visualisation of timelines and locations.

Large scale spatial context refers to specific locations on the surface of the Earth, their extent, and their names over time. Such information is treated by GIS systems using GPS reference coordinates and location inventories to treat these issues, respectively. The knowledge base keeps only the GPS association with user names of the location and their specification and classification in a given hierarchy or regions and countries; in Mingei GeoNames (https://www.geonames.org/) are used. The stored data can be presented in a variety of ways that show whether a place is neighbouring to the sea, up in the mountains, or an island. This is important for the presentation of craft context, as it better explains trade, resources and possible threats. At the same time, GIS visualisations is a way to relate to neighbouring, complementary destinations. In Mingei, we utilise the stored GIS data to create customised HC narrations that can be provided through popular media to a wide audience. Once encoded in GPS coordinates, the represented locations can be exported and integrated into any GIS system. In this way, the reconstructions produced can be integrated into the GIS platform of choice. In Figure 13 (right), the import of two locations in the OpenStreetMap [42] system is illustrated, for two locations in Krefeld, Germany.

The presentation of rural and environments often requires more specialised presentation. Denser spatial data exports of data and environment digitisations can be provided in the form of Virtual Reality (VR), with places of interest and events that occurred at particular locations to be added to the map. This way, each location can be enriched with any type of curated digital content (i.e., 3D reconstructions, historical information, video data, etc.) that have been included in the knowledge base. In Figure 14, two ways of presenting such data are proposed, for the island of Chios, Greece. The first is a projection room where overviews of the environment can be interactively presented from aerials trajectories. The second is a mobile application that presents the island in a 3D geophysical map. A third, more conventional way is provided by a custom VR viewer implemented in unity3D that allows the user to perform an indoors and outdoors virtual walkthrough inside the structures of Figure 15.

A piece of orientation material is a glossary of terms that associates triplets of terms, definitions, and visualisations from the $\mathrm{CI}$ representation. As a document, a glossary increases the value of a visit as pertinent concepts can be communicated prior to it and serves as a reminder after it. Several games and activities stem from this verbal, semantic, and visual association of content that is not necessarily restricted to the context of crafts. In this case, the important feature for sustainability relates to the capacity of communities creating and content upon their own capacities. Such glossaries are generated from the $\mathrm{CI}$ representation dynamically, using appropriate queries, in retrieving context in the appropriate language and visual context. The example in Figure 16, presents elements of a glass glossary. 

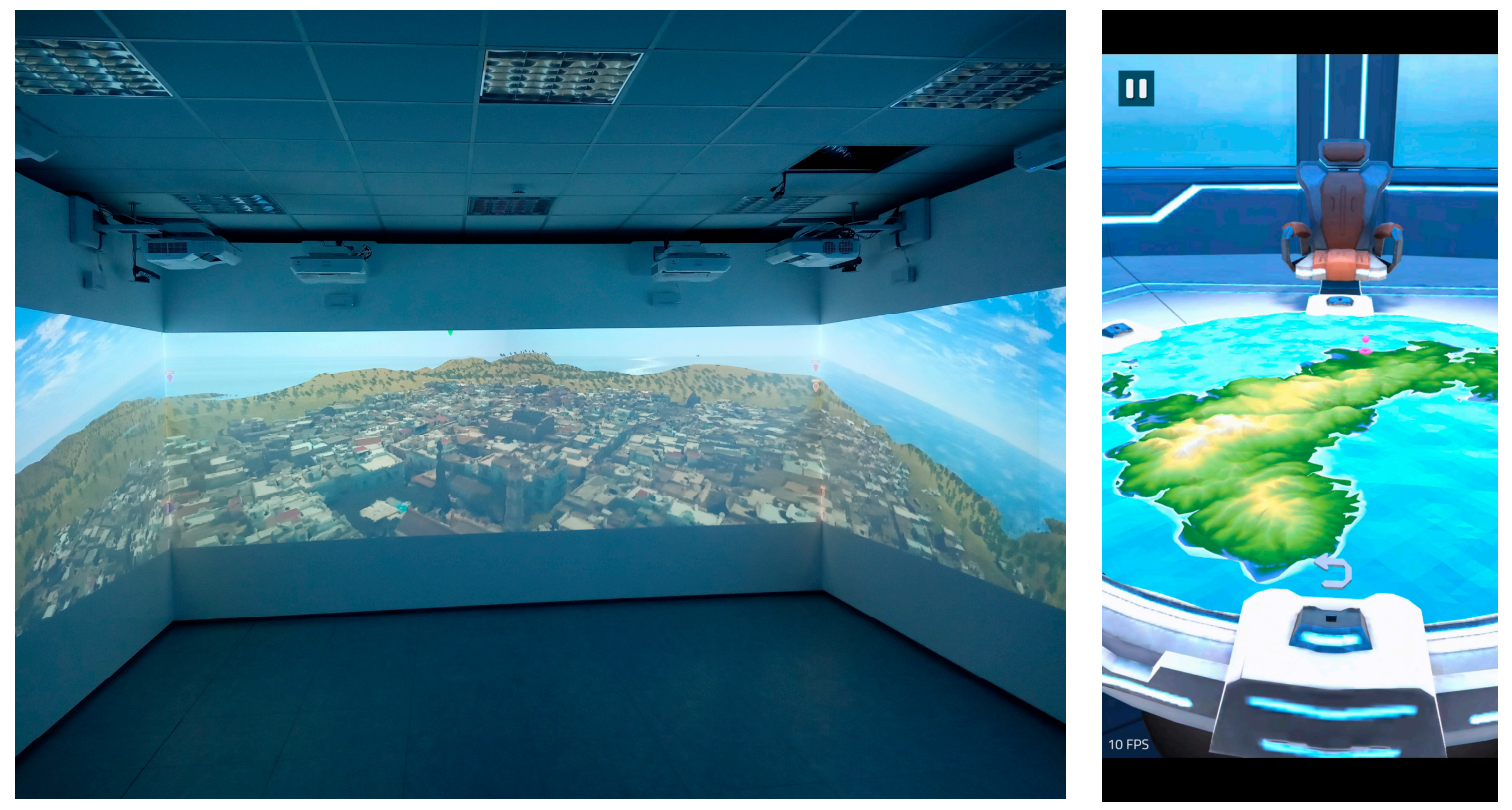

Figure 14. Immersive and VR presentation of geophysical context.
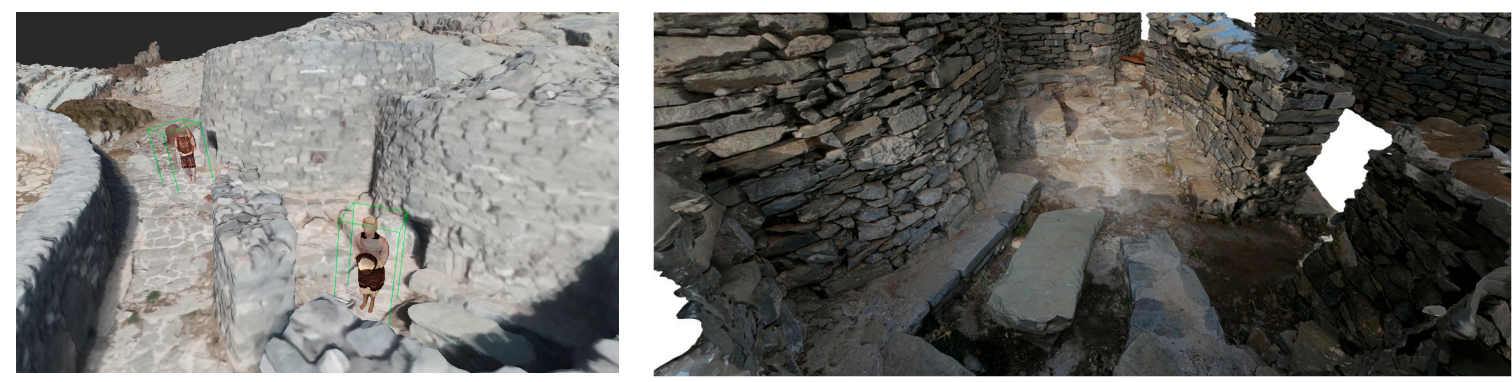

Figure 15. Virtual tour at animal husbandry settlements.

Craft gestures that include tool manipulation are visualised and trained in an educational context. The following example, in Figure 17, is a simple training scenario where the user is tasked to grasp a tool, using VR controllers and use in this case a hammer, a nail, and a wooden surface. The structure of the training scenario is broken down into smaller sub-tasks. The user is asked to pick up the tool. Performing this action equips the tool in the virtual hand, and the tool then follows the movement of the VR controller. Afterwards, a training animation activates which indicates how the tool is to be used, in this case how the hammer should be moved to hammer the nail down. The user is then asked to duplicate the indicated motion using the VR controller. Once the nail reaches the maximum depth, the training scenario is completed. This training scenario was created leveraging the ovidVR SDK, developed by ORamaVR [43].

MotiVo [44] is an interactive system that simplifies the process of motion visualisation by offering a number of visualisation tools as integrated components to an interactive system and provides insightful and visually pleasant results requiring minimum expertise and knowledge from the user (see Figures 18 and 19). A separate tool is used for presenting glass making processes in VR (see Figure 20). Using those tools, motion is visualised by parameters, such as the blending of key poses of activity, the visualisation of motion trajectories, the application of image filters to visualisations and their $3 \mathrm{D}$ and 2D combinations so as to create hybrid depictions of motion. 


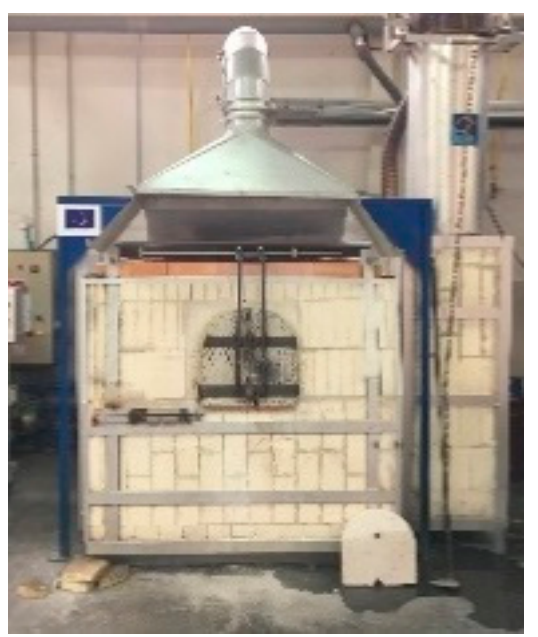

(a) Furnace: An enclosed structure for production and application of heat. Uses: Melting, maintaining, reheating, and annealing glass or glass objects

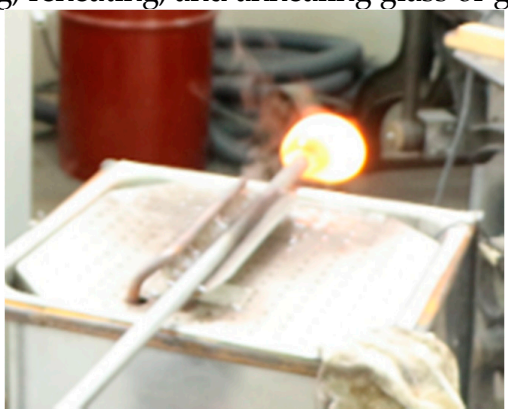

(b) Gather, or gob: A mass of molten glass collected at the end of a blowpipe, pontil, or gathering

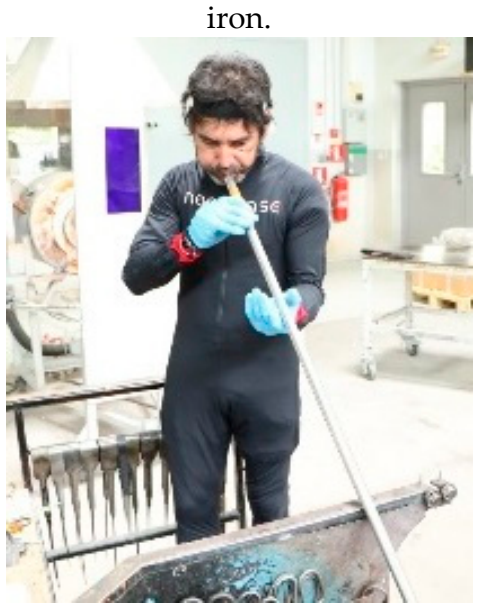

(c) Blowing: The technique of forming an object by inflating a gather or gob of molten glass on the end of a blowpipe.

Figure 16. Elements from the glass dictionary.
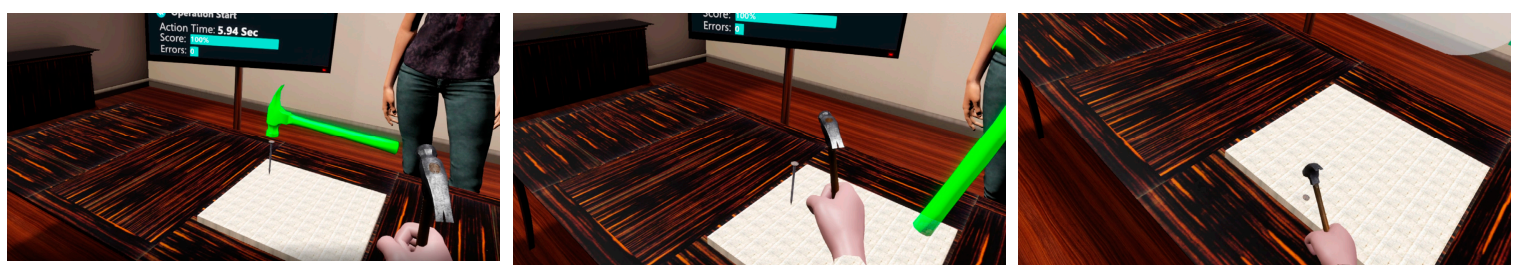

Figure 17. VR training tutorial for hammering a nail. Left: Tutorial animation indicating recommended motion, Middle: Movement the equipped tool into position for usage. Right: Hammering a nail. 

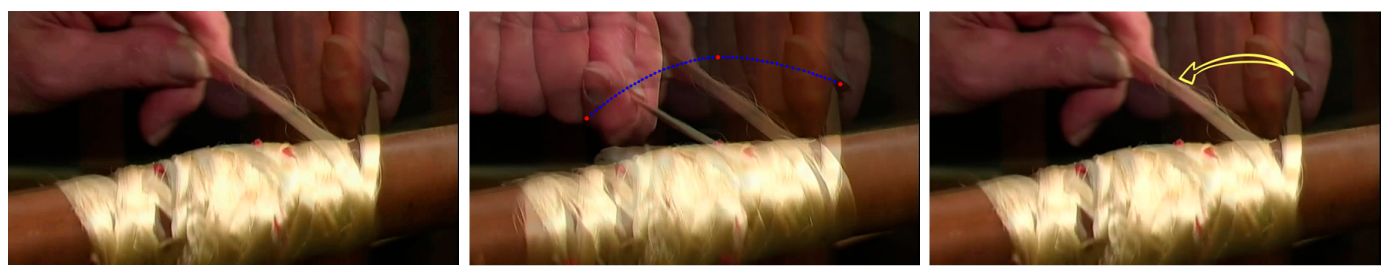

Figure 18. Authoring of gesture visualisations using MotiVo.
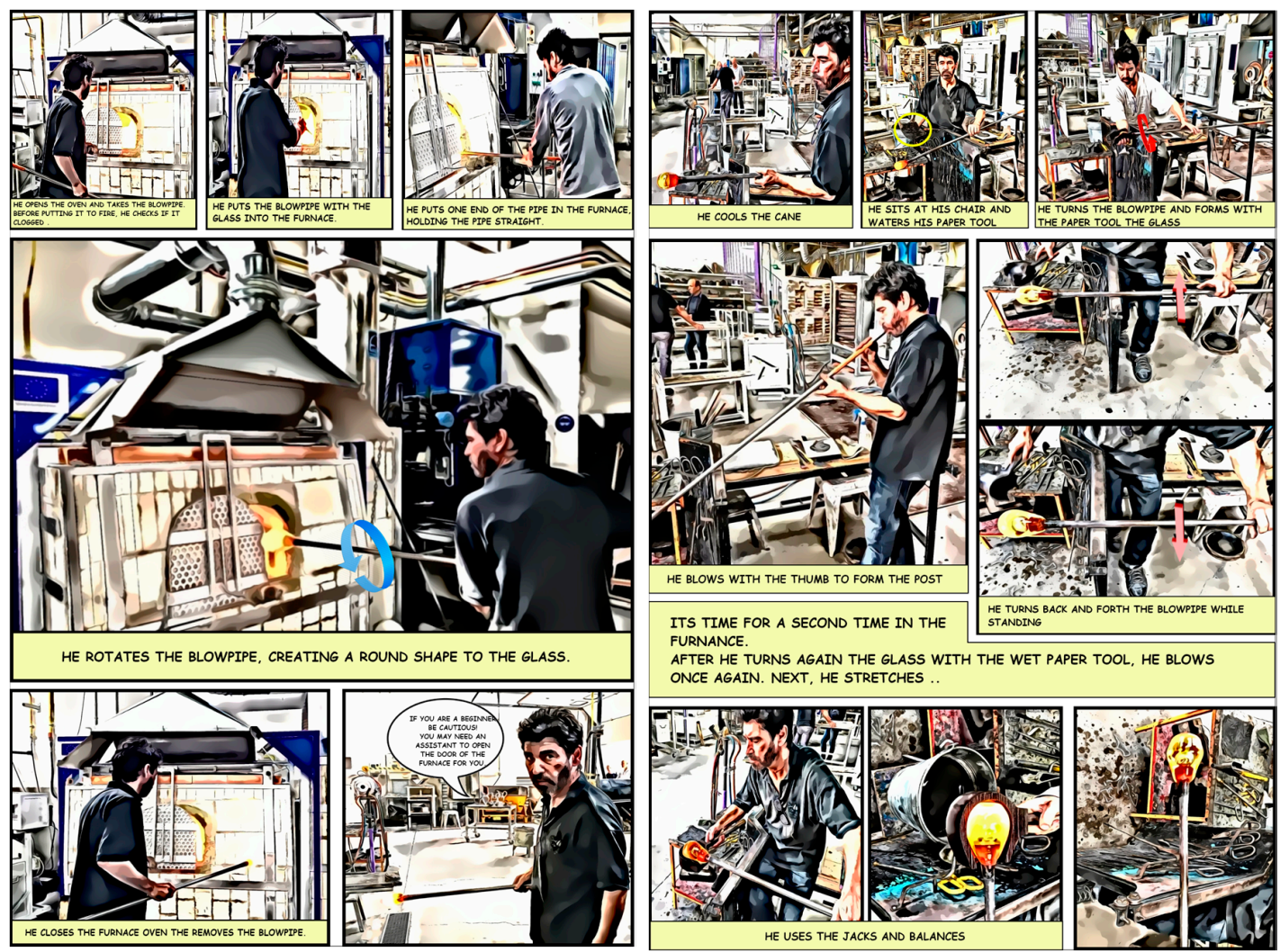

Figure 19. Authoring of illustrated instructions with MotiVo.
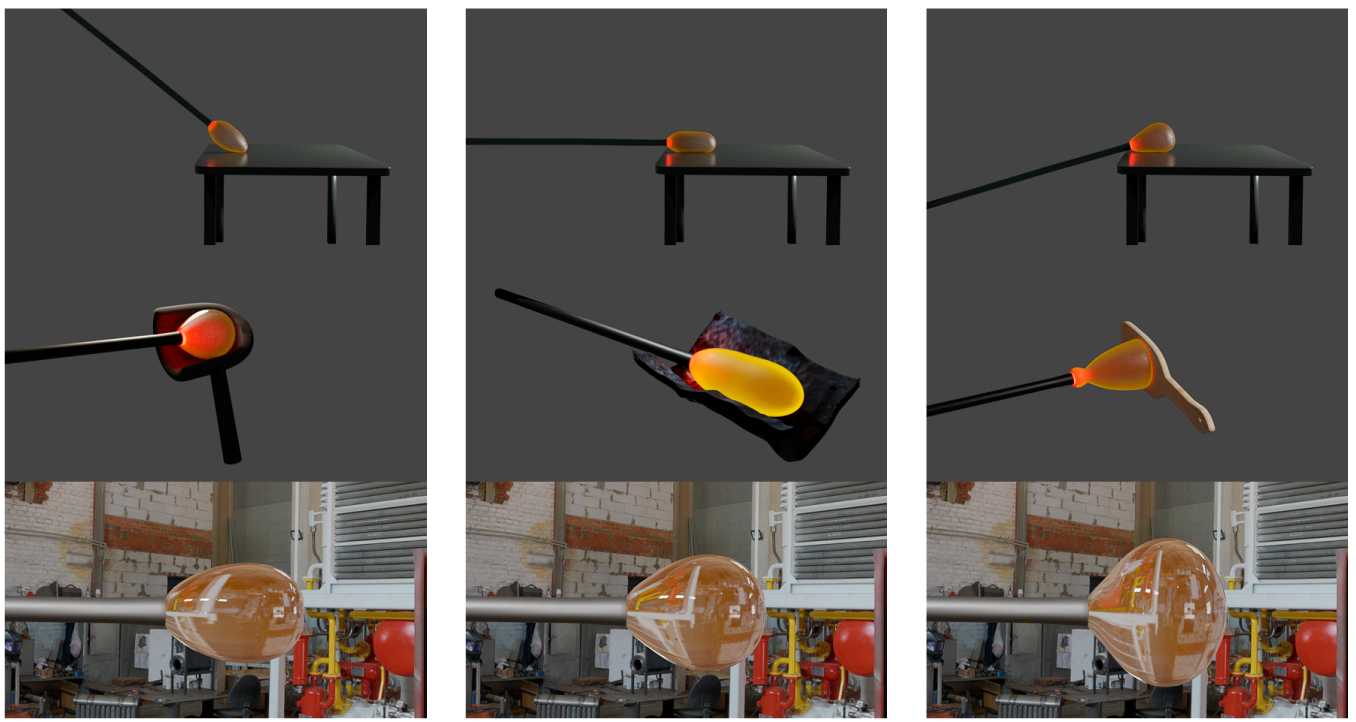

Figure 20. Computer-aided, VR presentation of glass making processes from [45]. Top: Marvering. Middle: Shaping. Bottom: Illustration of the glass deformation during glass blowing. 


\section{Use Cases}

\subsection{Margarites}

The island of Crete in Greece is an internationally popular tourist destination. The majority of tourism concentrates on the seaside locations as the majority of the tourism sector in Crete concentrates of sun-and-sea tourism. At the same time, the mountains of Crete host traditions that cover more than a millennium. Some of the most identifying elements of Cretan culture originate from the region of Psiloritis a UNESCO Global Geopark [46]. The village of Margarites is a simple to reach, mountainous and off-centre destination at this park, at an altitude of about $300 \mathrm{~m}$, within the region of this park and is one of the largest pottery centres in Greece.

Pottery is one of the oldest human crafts, originating before the Neolithic period, practiced by elemental materials and processes: Earth, water, and fire. Old and new techniques coexist in the 20 some pottery workshops and showrooms of the village, where traditional and modern pottery is produced. At Margarites, pottery tradition dates from the Neolithic period and is highly-relevant to the ample reserve of clay found at its rural surroundings at the mountain of Psiloritis.

Mingei encompasses an action towards cultural tourism relevant to traditional crafts and their participatory promotion and transmission. In collaboration with local policy makers and businesses, a tourism network is planned that involves participation in workshops and seminars with more than a day of the duration. Of relevance to the proposed work are pottery workshops held for students and visitors. Visitors can view and participate in a demonstration of traditional pottery. An initiation, educative experience into pottery was designed based on the modelling of traditional pottery in steps, showing how raw earth materials are transformed into pottery articles (see Figure 21). The process is modelled in five steps for which descriptions and educational videos are provided. The concept is showcased in a webpage (http://www.mingei-project.eu/pottery-experience-keramion/) [47].

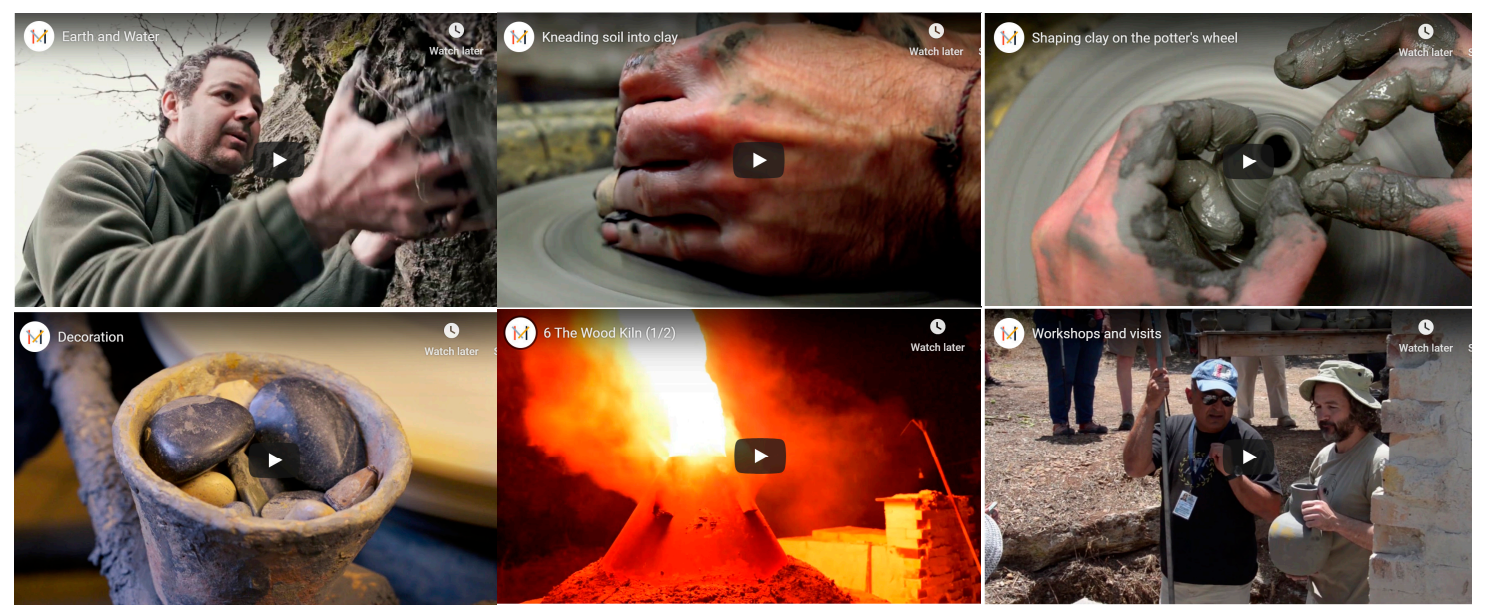

Figure 21. The basic steps of pottery making.

Nearby Locations of Interest are showcased to increase the value of a visit, such as the ancient city and archaeological museum of Eleutherna, as well as routes at the Psiloritis UNESCO Global Geopark, transhumance, the seasonal droving of livestock along migratory routes [33] as well as sample traditional Cretan cuisine is an expression of the Mediterranean diet [48].

\subsection{Chios}

In the south of the Greek island of Chios, a single type of tree has shaped not only the local trade and culture, but even the built environment. The tree does not grow anywhere else in the world and its resin, mastíha, is harvested to be used for a variety of uses, from skincare to medicinal and culinary use. Chios is well known throughout the world for the cultivation of mastic trees, since the 
antiquities. The know-how of cultivating, collecting, and processing mastic on the island of Chios is on the "Representative List of ICH of Humanity" of UNSECO [49].

Mastic is a natural product collected from the bark of mastic trees, which exclusively flourish in the southern part of the island, and due to this fact, the medieval settlements in that area are called "Mastichochoria" (Mastic Villages). These settlements date back to the Byzantine Era, and they are heritage monuments. During the Genoese occupation (1346-1566), the mastic cultivation becomes progressively systematic, and 22 mastic villages are founded in the southern part of the island aiming mainly at exploiting mastic, as a monopolistic product. The villages were not visible from the sea, and their layout resembles a fortress, designed to protect the inhabitants from the frequent incursions in the Aegean Sea.

Latin architects, engineers and contractors had local farmers build the villages, according to the Italian architectural model of that era. In the centre of each settlement, there was a tall rectangular tower, around of which there were houses very close to each other. The walls of the houses located circumferentially of the village formed an external wall with a cylindrical turret with crenellations on each corner to avoid invaders who tried to approach the centre of the village by confusing them. This architectural draft gave masters the opportunity to close the gates in order to protect the area and simultaneously keep their subjects under control.

To illustrate this geographical context, an overview was preferred. Aerial images were acquired via a UAV. The subjects are large building complexes. The building structure is challenging, due to the complex, rural city planning and fortification. Corresponding reconstructions from villages Pyrgi, Mesta, Olympoi and Elata are shown in Figure 22. Two figures are provided for each village; an annotated top view and a close-up near to its central building. The figures are annotated as follows; red lines represent densely constructed houses which formed continuous external walls, green represents the location of the central tower where mastic was stored, and blue circles represent rescued turrets. Note that in later years, the towers in Mesta and Elata were replaced by churches. Detailed videos from the reconstructions are provided in the Supplementary Material.

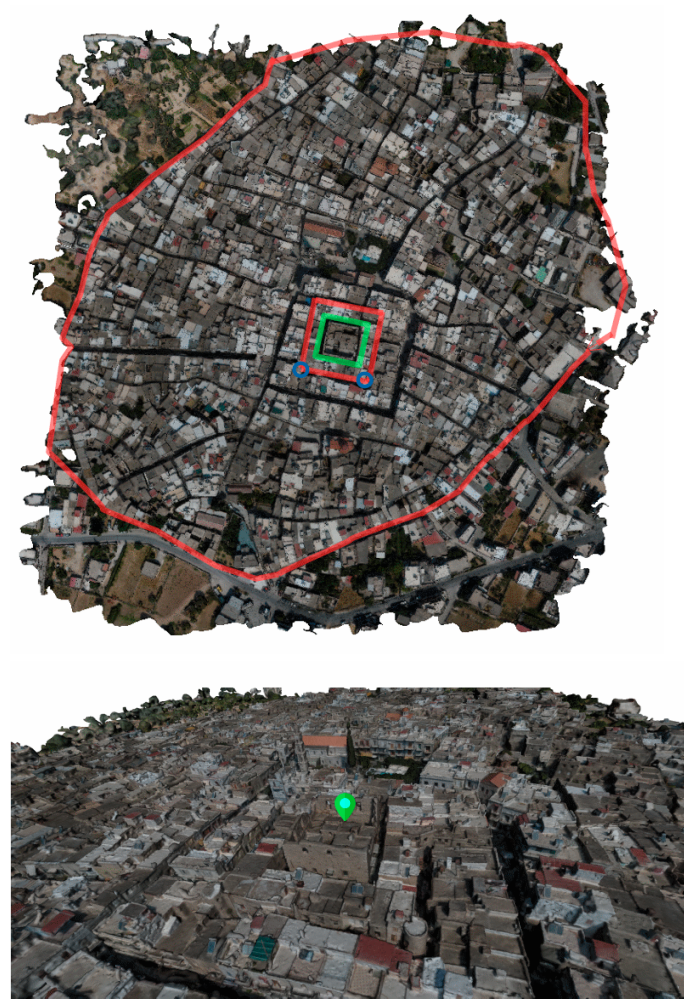

(a) Pyrgi
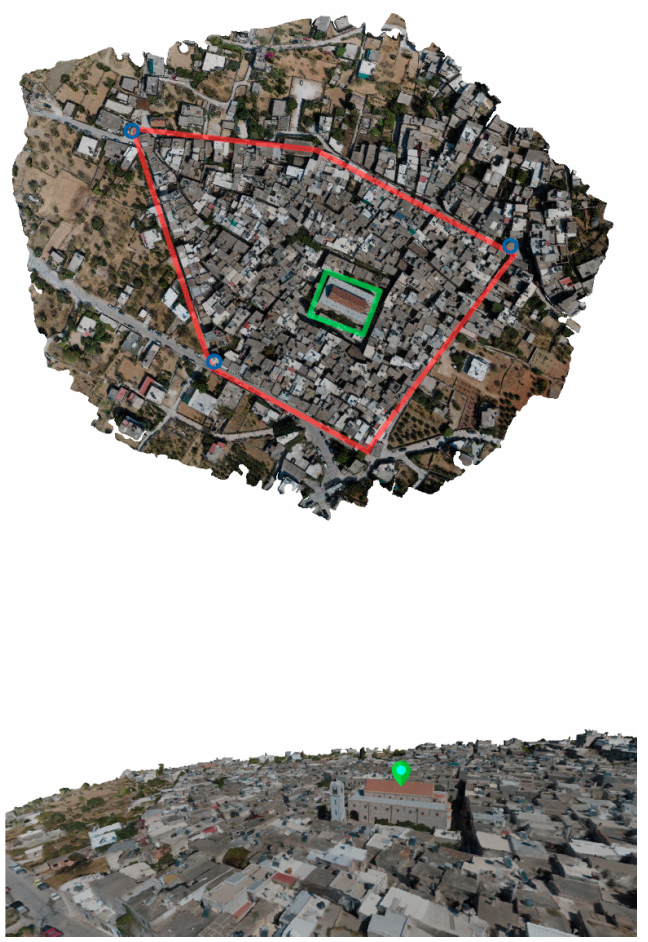

(b) Mesta

Figure 22. Cont. 

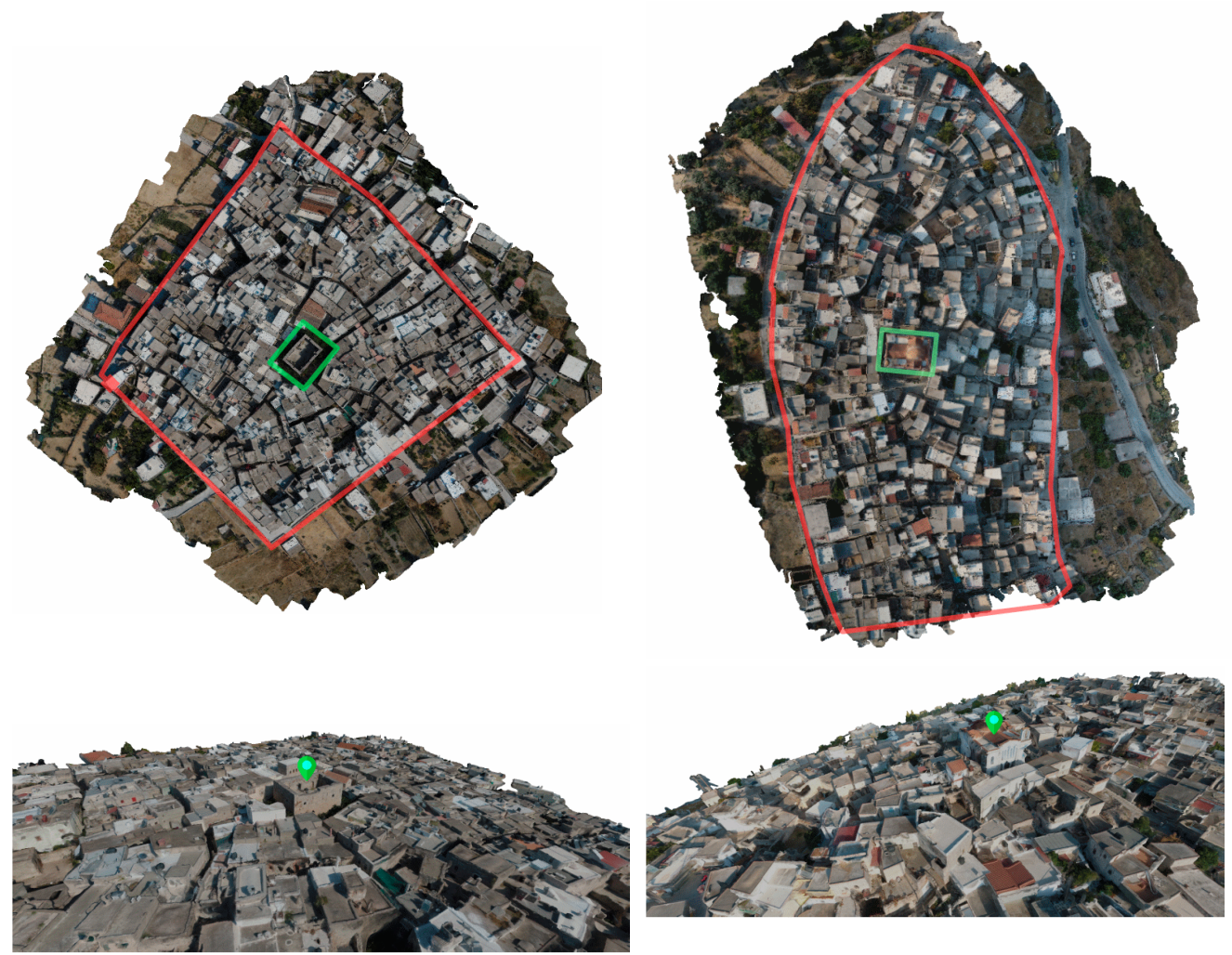

(c) Olympoi

(d) Elata

Figure 22. "Mastichochoria" reconstructions.

The crafts related to mastic contain both outdoor and indoor activities that take place around the house and in a centralised location. Digital representations of machines (i.e., Figure 23) and traditional garments (i.e., Figure 24) have been acquired from the Mastic Museum at Chios. These are utilised in a VR application (see Figure 25) that has been developed that allows the exploration of the natural environment from a terrestrial viewpoint. The terrain of the game was generated by importing the geophysical map into unity3D. Then flora and fauna were imported together with 3D reconstructions of villages. The concept of the game is to explore the island in different eras and acquire knowledge about the cultivation and trade of mastic in each one, through a mission-oriented approach. Digitised rural environments are also important to the realism of such application.
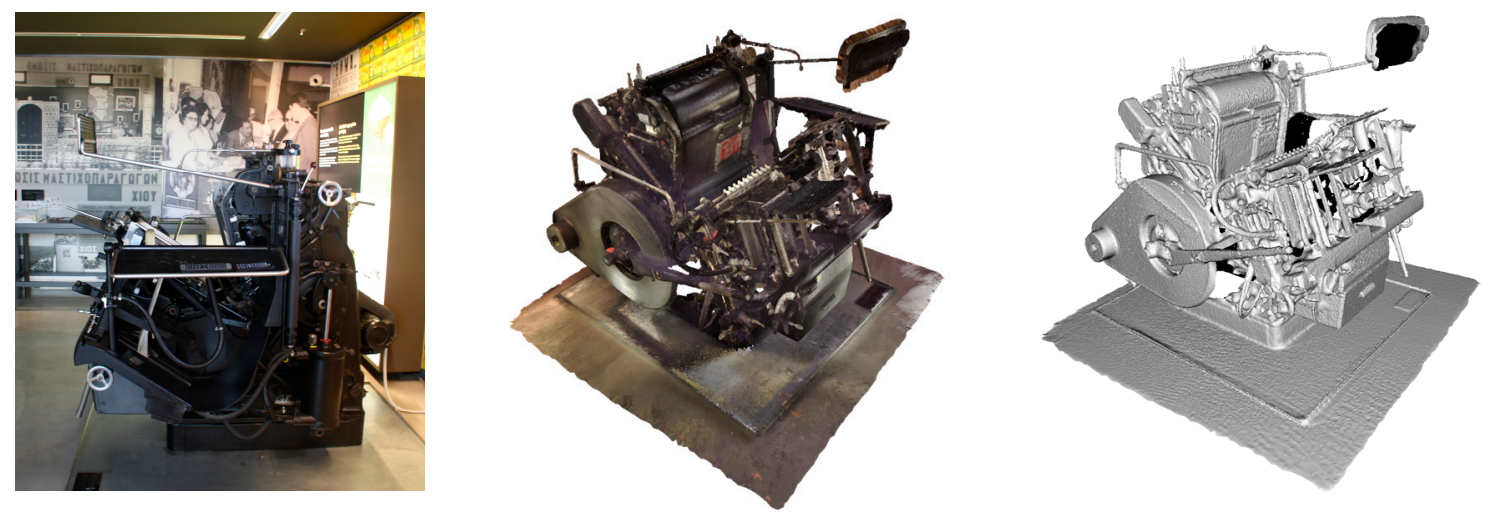

Figure 23. Digitisation of Machines for mastic processing. 

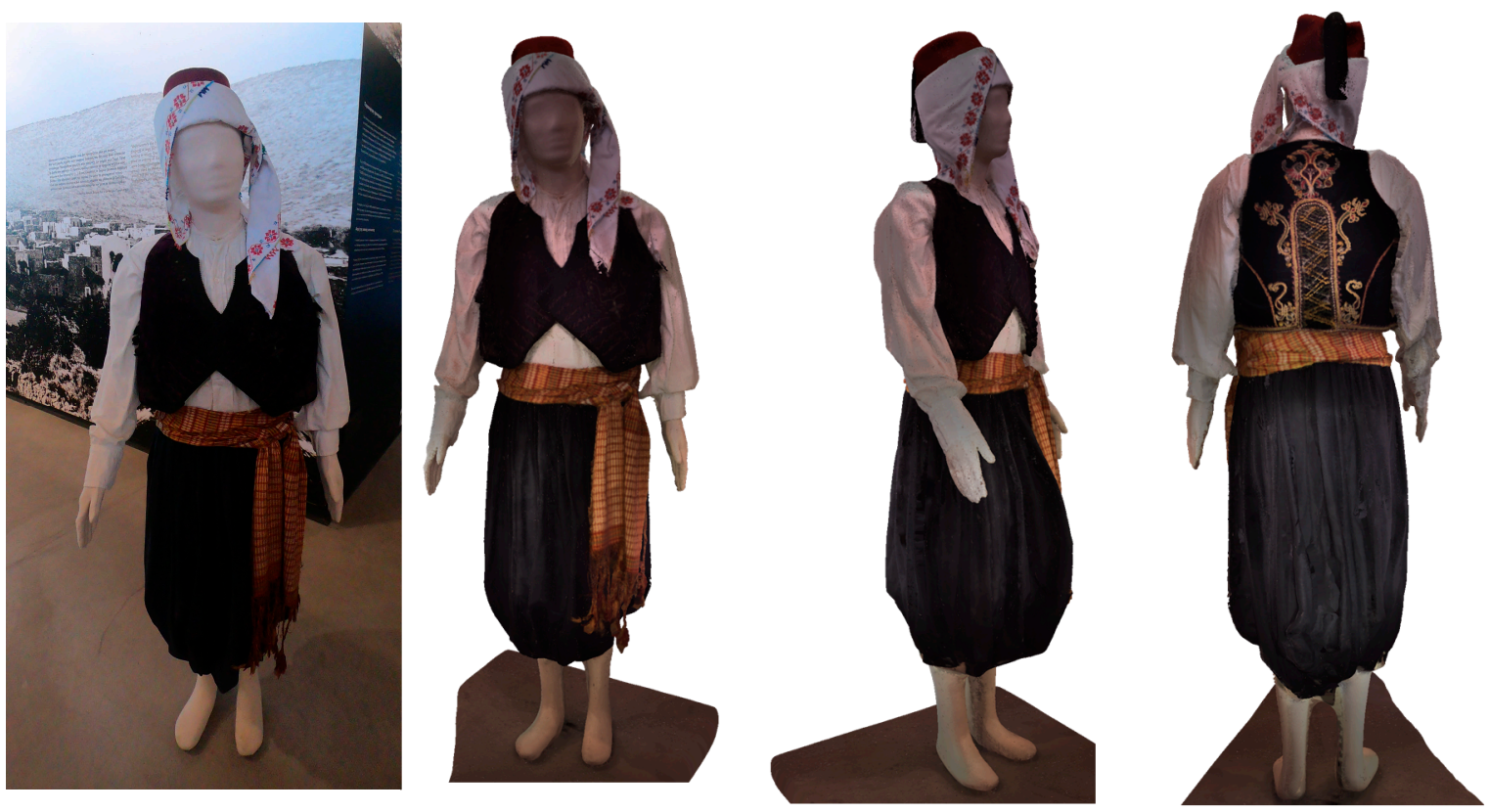

Figure 24. Digitisation of traditional garments of Chios.

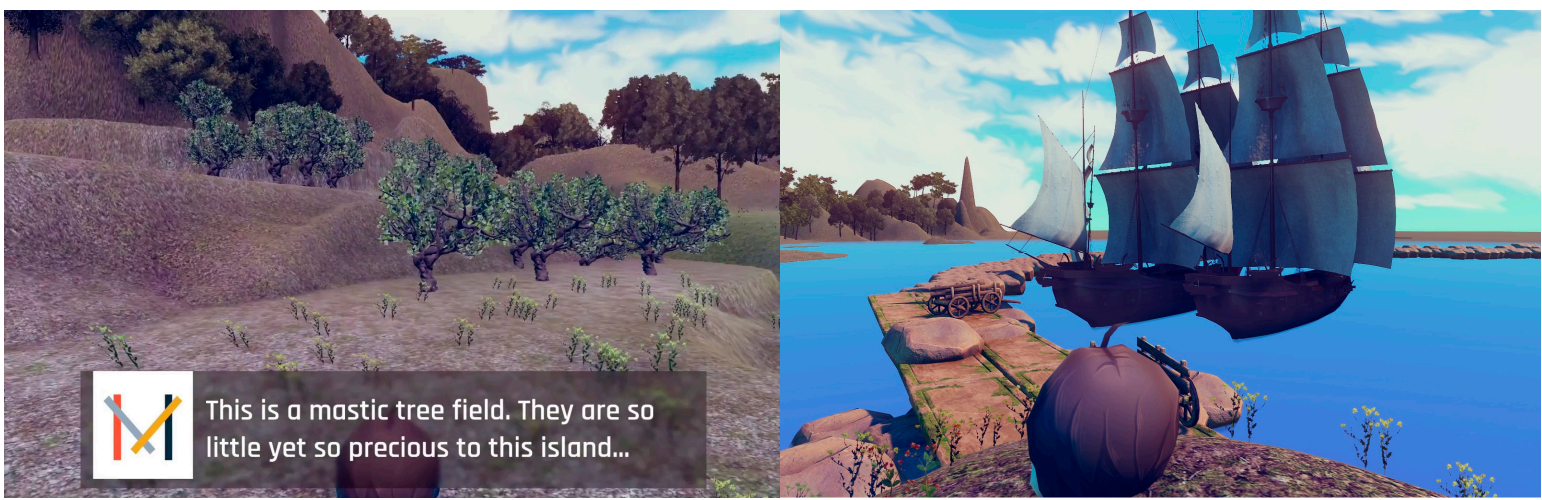

Figure 25. A VR application for the presentation of mastic trade and the exploration of the natural environment of Chios.

In Figure 26, a mobile application (a demonstration video of the application can be found here: https://youtu.be/JjOcxmrQ744) presents a thicker context introducing the traditional knowledge on how to induce incision on a mastic tree. This practice extends over a period of months, visiting the trees periodically. Of relevance are not only the direction and depth of the incision, but also the location on the step, the order, the density and the number and location of incisions per time of year. The technique and knowledge are presented in multiple ways, in a mobile application, presenting narratives associated through the "thick" CI representation. In this case, the number of incisions per time of year is relevant to slowly "wake" the tree in the winter and at its base. The order relates to the optimisation of production; traditionally, order is from bottom to top. Density, relates to an emic understanding of the craft, as it is associated with the intention of maximising production on the cost of the tree's well-being, as overharvesting is well known to damage the tree and eventually reduce next year's production. As such, the overly dense incision has a negative connotation. In Figure 26, a mobile application that simulated mastic cultivation is presented. In the example, the recommended motion to create an incision to a mastic tree, in order to collect its resin, is simulated. 

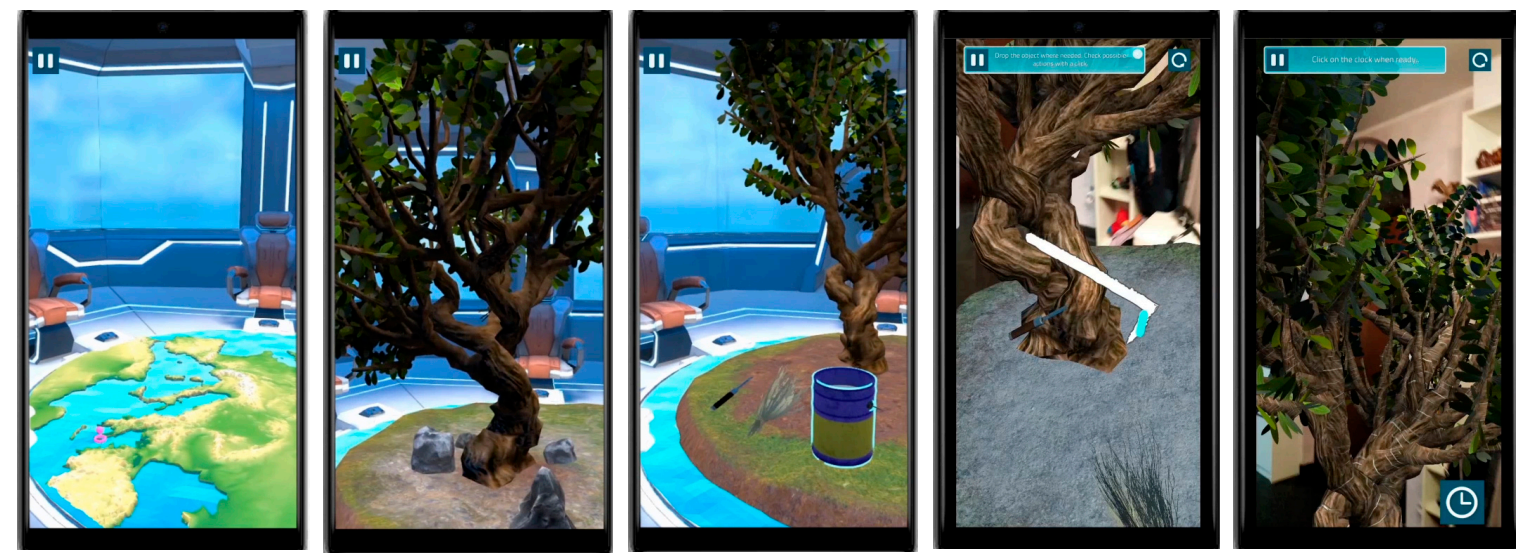

Figure 26. A mobile application that simulates the tasks of mastic cultivation.

\subsection{Krefeld}

Krefeld is a city in North Rhine-Westphalia, Germany, with a rich industrial and cultural history tied to silk and velvet weaving from the 1600s onwards. Krefeld, also known as the "Town like Silk and Velvet", is not a mainstream touristic destination; however, it has a lot to offer to visitors. In the context of the silk pilot in Mingei and through the collaboration with the Museum "Haus der Seidenkultur", a number of narratives have been formulated to support the storyline of the silk weaving craft in Krefeld. Such narratives include: Krefeld history from its origins to its transformation to the town of Silk and Velvet, Jacquard weaving, History of the Haus der Seidenkultur, understanding the craft of silk Jacquard weaving, ecclesiastical fabric weaving in Krefeld, ecclesiastical textiles, etc.

In Mingei, we are examining how these narratives can be used to support a thematic tourism use-case scenario with multiple exploitation opportunities. One of the examined methods is to utilise the silk weaving narratives to create customisable city tours. The vision is to provide a way for the stakeholder to create city tours customised to various target audiences, i.e., families, individual visitors, Rhine cruise visitors, etc. Using their mobile devices, the visitors will have access to a selection of rich $\mathrm{CH}$ digital content from the Mingei platform associated with the specific points of interest included in each tour, formulated in user-friendly multimodal presentations and experiences. The material can be used in the context of existing guided tours, of creating new tours, or even as a means of advertising to attract new visitors.

In this example, a city walk for Krefeld has been created. In each location of interest, a video and when required, a 3D reconstruction is associated with the accompanying online digital content. Figure 27 shows export of our modelled GIS information in the format of Google Maps (The Krefeld Silk City Tour Map can be found at the following link. The link to the associated videos are in the comments of each location: https://bit.ly/2SBPodn). In Figure 27, the 3D reconstruction of a weaver's house is shown along with the video narration. In the particular case, the 3D structure of the building is of interest to the craft, and therefore, its 3D reconstruction is shown. As explained in the accompanying video, the reason is its windows and the indoor light that they avail. Weaving is a craft that requires light and prior to electricity windows made it possible to work longer hours. For this reason, weaver's homes tended to have multiple windows on more than one face of the house. The route covers locations relevant to Krefeld's silk textile manufacturing history, in a recommended order. Each location is linked to a curated video narration that presents historical and cultural data about it. Building data that are relevant to the craft can be presented this way, such as the relevance of windows and weaving in a temporal context.

More abstract and analytic knowledge, as well as the use of rare machines, can be presented with the use of VR. In Figure 28, a game explains the connection between the point paper design and the production of textile motifs (A demonstration of the game can be found here: https://youtu.be/-0ep8g A1u0). The game is designed to provide a better understanding of the artistic dimension of textile 
design and manufacturing. During the co-creation meetings and talks with stakeholders, an interesting issue was raised. The work of the various craftsmen to first create a design, to transform this to point paper and ultimately to punch the cards which control the Jacquard unit whilst weaving is frequently overlooked or barely even recognised by the visitors to the museum. The first craftsman draws a picture or motif in pencil; the point paper designer transfers this to the point paper using watercolours. This point paper design is then used to punch cards for the Jacquard loom. To highlight this feature, a new game has been designed, in which the user will be able to draw his/her own pattern and then punch a corresponding card in order to produce the final fabric and see the final textile production.

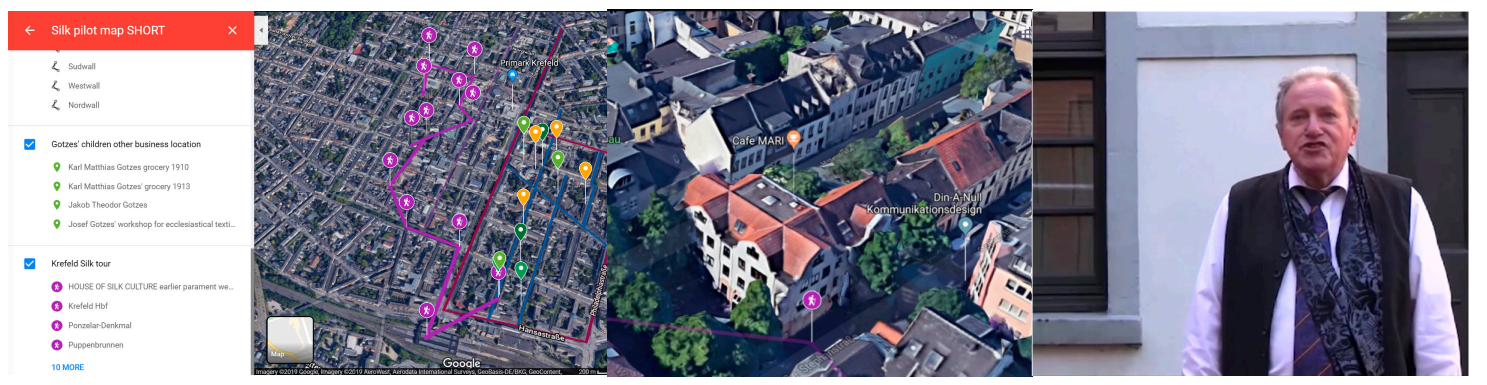

Figure 27. A weaver's house.
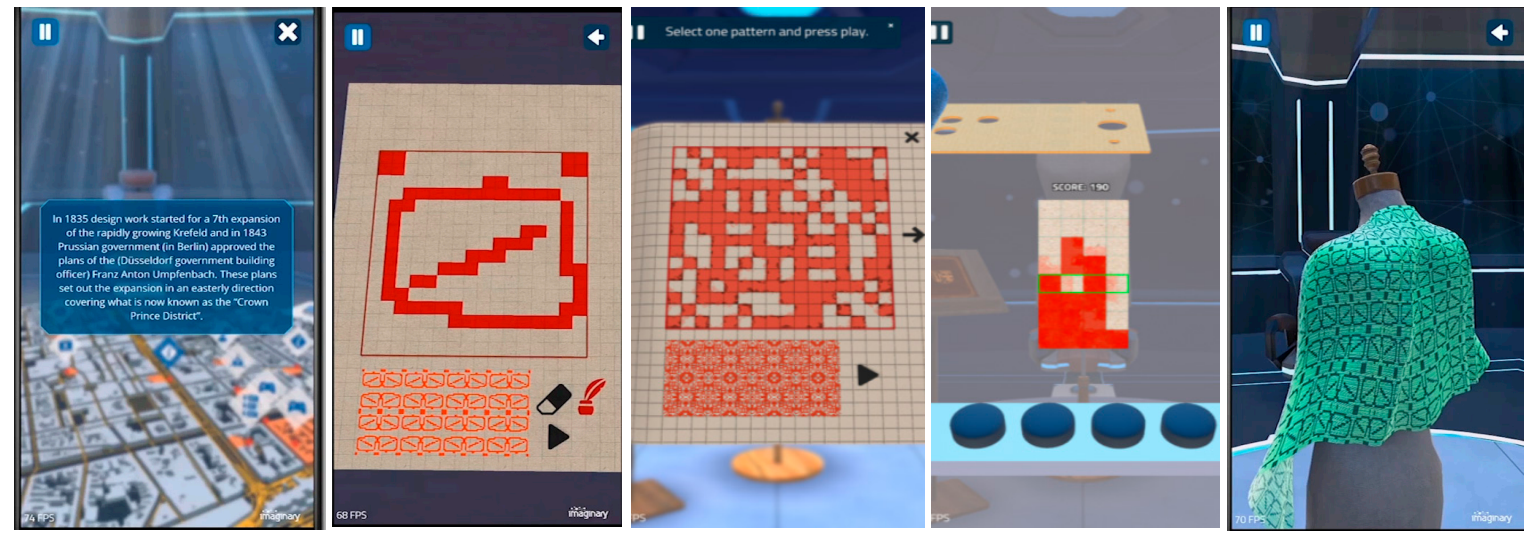

Figure 28. A mobile application that simulates point paper design, card perforation for the Jacquard attachment, and actual weaving and visualisation.

\section{Conclusions}

The contribution of culture in building traditional knowledge and skills, including local knowledge and cultural diversity, is the core of the Mingei project. Accurate representation of crafts and educational tool contribute to the transmission of local cultural values, knowledge, and skills.

Empowerment of local, rural communities is supported by simple digital tools in order to inventorize and represent knowledge, in an inclusive and participatory fashion with communities of practitioners. The benefit of using simple technologies contributes to the building of the capacity building, for the preservation of local culture.

To provide relevant educational content and engaging experience for uses prior, during, and after a cultural tourism visit. The promising possibilities of thematic tourism emphasize the importance of the preservation and representation of heritage crafts.

In compliance with global, collective efforts, the Mingei approach is oriented towards satisfying the four basic axes of the 2030 UNESCO agenda [50]. Applications focus on the natural environment and its resilience, presenting the role of natural resources, history, and tradition in the shaping of local cultural heritage. The applications are oriented toward safeguarding and sustainable management, by the inclusion of traditional knowledge in cultural tourism services. Applications present traditions, 
memories, and values of past generations in local craft communities, obtained through defining events of prosperity or tension in their history. The recite of difficulties of past generations through stories and tradition, tends to increase the sense of obligation towards future generations in terms of environmental protection [51]. In the context of the natural environment and $\mathrm{ICH}$, this corresponds to safeguarding and sustainability of the pertinent environmental resources. Prosperity and livelihood are served by the contribution of culture in inclusive and sustainable economies, by generating income and employment in cases where the number of practitioners is declining, as well as stimulating revenue through cultural goods, services, and enterprises. In other words, Mingei strives to preserve interest sustainably, and thus, continuation of the practice that is the main prerequisite of ICH preservation.

Supplementary Materials: The following are available online at http://www.mdpi.com/2071-1050/12/4/1461/s1. The Videos can be found at https://zenodo.org/record/3679130\#.X1EPHkoRVPY. Webpage S1: The demonstration web page for pottery at Margarites, Video S2: The mastic application demonstration, Video S3: The Krefeld game demonstration, Webpage S4: The Krefeld Silk City Tour Map, Webpage S5: Textile magnification demonstrator, Video S6: 3D reconstruction of Sleeping Female Figure, Video S7: The 3D rural reconstruction videos of mastic museum and mastic villages villages Pyrgi, Olympoi, Mesta, Elata. Reconstructions 3D: (1) Sleeping Female Figure. The sculpture can be viewed in the following link: https://youtu.be/1hyTKpSIIUw; The sculpture was recorded on November 2019, at Athens, Greece. It is the "Sleeping Female Figure" (1877), by sculptor Yannoulis Chalepas (1851-1938). (2) Mastic villages. The 3D rural reconstruction videos can be found here: Mastic Museum https://youtu.be/Yw-xQY1KxQs; Pyrgi https://youtu.be/15NcRlg3360; Olympoi https://youtu.be/adPDVx7RGVc; Mesta https://youtu.be/9xNlrGSBfIE; Elata https://youtu.be/wUGY3aoP6y4.

Author Contributions: Conceptualization, X.Z. and N.P. (Nikolaos Partarakis); methodology, X.Z., V.B., D.M., C.M., I.A., S.N.; software, A.Q., N.P. (Nikolaos Partarakis), N.S., I.S., A.C., N.P. (Nikolaos Patsiouras), P.D., E.K., E.S., V.N., A.R.; validation, I.A., S.N.; formal analysis, C.M.; investigation, C.B., I.N.-J., U.D., H.H., A.D., D.K.; resources, M.F.; data curation, C.B., A.D., D.K., M.F., A.P.; writing—original draft preparation, X.Z.; writing-review and editing, G.G.; visualization, N.P. (Nikolaos Patsiouras), E.S., A.R., E.M.; supervision, X.Z.; project administration, X.Z. All authors have read and agreed to the published version of the manuscript.

Funding: This work was supported by the European Commission Horizon 2020 Project, Grant No. 822336 and the Foundation for Research and Technology Hellas-Institute of Computer Science (FORTH-ICS) internal RTD Programme 'Ambient Intelligence and Smart Environments'.

Acknowledgments: The authors thank Dieter Blatt, Guenther Oehms, Manfred Weisters and Dieter Brenner and the Association of Friends Haus der Seidenkultur for their contribution to the video recordings. Authors thank Giorgis Dalambelas for the pottery demonstrations.

Conflicts of Interest: The authors declare no conflict of interest.

\section{References}

1. UNESCO. Traditional Craftsmanship. Available online: https://ich.unesco.org/en/traditional-craftsmanship00057 (accessed on 16 January 2020).

2. KEA European Affairs. The Economy of Culture in Europe: A Study Prepared for the European Commission (Directorate-General for Education and Culture). Available online: https://ec.europa.eu/assets/eac/culture/ library/studies/cultural-economy_en.pdf (accessed on 12 February 2020).

3. UNESCO. Creative Economy Report: Widening Local Development Pathways. Available online: https: //unesdoc.unesco.org/ark:/48223/pf0000224698 (accessed on 12 February 2020).

4. KEA European Affairs. The Impact of Culture on Creativity. Available online: https://europa.eu/capacity4dev/ file/18617/download?token=Fzta9pMB (accessed on 12 February 2020).

5. TERA Consultants. Building a Digital Economy: The Importance of Saving Jobs in the EU's Creative Industries. Available online: https://euipo.europa.eu/ohimportal/documents/11370/71142/Building+a+Digital+Economy, +the+importance+of+saving+jobs+in+the+EUs+creative+industries (accessed on 12 February 2020).

6. Kreidi, J. Crafts and Tourism: UNESCO Action. In Proceedings of the First International Congress on Tourism and Traditional Crafts, Riyadh, Saudi Arabia, 7-14 November 2006.

7. OECD Annual Report 2009. Available online: https://www.oecd.org/newsroom/43125523.pdf (accessed on 16 January 2020).

8. Du Cros, H. UNWTO Study on Tourism and Intangible Cultural Heritage: Examples of Best Practices from Australia, Indonesia, Thailand and Vanuatu. 2011. Available online: https://www.e-unwto.org (accessed on 12 February 2020). 
9. Rennick-Egglestone, S.; Brundell, P.; Koleva, B.; Benford, S.; Roussou, M.; Chaffardon, C. Families and mobile devices in museums: Designing for integrated experiences. J. Comput. Cult. Herit. (JOCCH) 2016, 9, 11. [CrossRef]

10. ETH Library's Best Practices in Digitization. Available online: https://www.library.ethz.ch/en/ms/DigiCenter/ Best-Practices-in-Digitization (accessed on 16 January 2020).

11. Brosseau, K.; Choquette, M.; Renaud, L. Digitization Standards for the Canadian Museum of Civilization Corporation. Available online: http://museumsassn.bc.ca/wp-content/uploads/2015/01/smcc_numerisationcmcc_digitization-eng.pdf (accessed on 16 January 2020).

12. CARLI Digital Collections Users' Group; CARLI Created Content Committee. Guidelines for the creation of digital collections, Consortium of Academic and Research Libraries at the University of Illinois. Available online: https://www.carli.illinois.edu/sites/files/digital_collections/documentation/guidelines_for_video.pdf (accessed on 16 January 2020).

13. 3D-ICONS Project. Guidelines \& Case Studies. Available online: https://pro.europeana.eu/files/Europeana Professional/Projects/Project_list/3D-ICONS/Deliverables/3D-ICONS\%20Guidelines\%20and\%20Case \% 20Studies.pdf (accessed on 16 January 2020).

14. MINERVA Project. Good Practices Handbook. Available online: https://www.minervaeurope.org/structure/ workinggroups/goodpract/document/goodpractices1_3.pdf (accessed on 16 January 2020).

15. Europeana Collections. Available online: https://www.europeana.eu/ (accessed on 16 January 2020).

16. Doerr, M.; Ore, C.-E.; Stead, S. The CIDOC conceptual reference model: A new standard for knowledge sharing. In Proceedings of the Tutorials, Posters, Panels and Industrial Contributions at the 26th International Conference on Conceptual Modeling, Auckland, New Zealand, 5-9 November 2007; Volume 83, pp. 51-56.

17. I-Treasures Project. Available online: https:/ec.europa.eu/digital-single-market/en/blog/i-treasurescapturing-intangible-cultural-heritage-and-learning-rare-know-how-living-human (accessed on 17 January 2020).

18. Modul-Dance Project. Available online: http://www.modul-dance.eu/ (accessed on 17 January 2020).

19. Dance Project. Available online: http://dance.dibris.unige.it/ (accessed on 17 January 2020).

20. European Theatre Lab. Available online: https://www.europeantheatrelab.eu/ (accessed on 17 January 2020).

21. Terpsichore Project. Available online: http://terpsichore-project.eu/ (accessed on 17 January 2020).

22. Geijer, A. A History of Textile Art; Pasold Research Fund: London, UK, 1979.

23. Hecht, A. The Art of the Loom; British Museum Press: London, UK, 1989.

24. Donkin, L. Crafts and Conservation: Synthesis Report for ICCROM. Available online: https://www.iccrom. org/publication/crafts-and-conservation-synthesis-report-iccrom (accessed on 17 January 2020).

25. Costin, C.L. Introduction: Craft and social identity. Archeol. Pap. Am. Anthropol. Assoc. 1998, 8, 3-16. [CrossRef]

26. UNESCO. Guidelines for the Preservation of Digital Heritage. Available online: https://unesdoc.unesco.org/ ark:/48223/pf0000130071 (accessed on 17 January 2020).

27. Fotopoulou, S.V.; Drinis, Y.N.; Bazini, E.; Fakiola, M. Rural Space as Cultural Heritage. Available online: http://ayla.culture.gr/wp-content/uploads/2018/10/1st_S_S_2018_Rural_Space_as_Cultural_Heritage_ WebENG.pdf (accessed on 16 January 2020).

28. Partarakis, N.; Zabulis, X.; Antona, M.; Stephanidis, C. Transforming Heritage Crafts to engaging digital experiences. In Visual Computing for Cultural Heritage; Liarokapis, F., Voulodimos, A., Doulamis, N., Doulamis, A., Eds.; Springer: Basel, Switzerland, 2019.

29. Zabulis, X.; Meghini, C.; Partarakis, N.; Kaplanidi, D.; Doulgeraki, P.; Karuzaki, E.; Stefanidi, E.; Evdemon, T.; Metilli, D.; Bartalesi, V. What Is Needed to Digitise Knowledge on Heritage Crafts. Memoriamedia Review. 2019. Available online: https://review.memoriamedia.net/ (accessed on 12 February 2020).

30. Stefanidi, E.; Partarakis, N.; Zabulis, X.; Zikas, P.; Papagiannakis, G.; Thalmann, N.M. TooltY: An approach for the combination of motion capture and 3D reconstruction to present tool usage in $3 \mathrm{D}$ environments. In Intelligent Scene Modelling and Human Computer Interaction; Thalmann, N.M., Zheng, J., Eds.; Springer: Basel, Switzerland, 2020.

31. Zhang, J.; Wu, Q.; Zhang, J.; Shen, C.; Lu, J.; Wu, Q. Heritage image annotation via collective knowledge. Pattern Recognit. 2019, 93, 204-214. [CrossRef] 
32. Su, X.; Sperlì, G.; Moscato, V.; Picariello, A.; Esposito, C.; Choi, C. An edge intelligence empowered recommender system enabling cultural heritage applications. IEEE Trans. Ind. Inform. 2019, 15, 4266-4275. [CrossRef]

33. UNESCO. Transhumance, the Seasonal Droving of Livestock Along Migratory Routes in the Mediterranean and the Alps. Available online: https://ich.unesco.org/doc/src/42975-EN.doc (accessed on 16 January 2020).

34. UNESCO. Art of Dry Stone Walling, Knowledge and Techniques. Available online: https://ich.unesco.org/ doc/src/39132-EN.doc (accessed on 17 January 2020).

35. UNESCO. Tinian Marble Craftsmanship. Available online: https://ich.unesco.org/doc/src/30299-EN.doc (accessed on 16 January 2020).

36. Nansense Website. Available online: https://www.nansense.com (accessed on 17 January 2020).

37. Qammaz, A.; Argyros, A.A. MocapNET: Ensemble of SNN Encoders for 3D Human Pose Estimation in RGB Images; BMVA Press: Guildford, UK, 2019.

38. Panteleris, P.; Oikonomidis, I.; Argyros, A. Using a single RGB frame for real time 3D hand pose estimation in the wild. In Proceedings of the 2018 IEEE Winter Conference on Applications of Computer Vision (WACV), Lake Tahoe, NV, USA, 12-15 March 2018; pp. 436-445.

39. ELAN Annotation Tool. Available online: https://tla.mpi.nl/tools/tla-tools/elan (accessed on 17 January 2020).

40. Zabulis, X.; Lourakis, M.I.; Stefanou, S.S. 3D Pose Refinement Using Rendering and Texture-Based Matching. In Proceedings of the International Conference on Computer Vision and Graphics, Warsaw, Poland, 5-17 September 2014; pp. 672-679.

41. Stefanidi, E.; Partarakis, N.; Zabulis, X.; Papagiannakis, G. An Approach for the Visualization of Crafts and Machine Usage in Virtual Environments. In Proceedings of the ACHI 2020 (The 13th International Conference on Advances in Computer-Human Interactions), Valencia, Spain, 22-26 March 2020.

42. Haklay, M.; Weber, P. Openstreetmap: User-generated street maps. IEEE Pervasive Comput. 2008, 7, 12-18. [CrossRef]

43. Orama, V.R. Available online: http://oramavr.com/ (accessed on 17 January 2020).

44. Rigaki, A.; Partarakis, N.; Zabulis, X.; Papagiannakis, G. An Approach Towards Artistic Visualizations of Human Motion in Static Media Inspired by the Visual Arts. In Proceedings of the ACHI 2020 (The 13th International Conference on Advances in Computer-Human Interactions), Valencia, Spain, 22-26 March 2020.

45. Schmid, E.T. Beginning Glassblowing; Glass Mountain Press: Bellingham, WA, USA, 1998.

46. Psiloritis UNESCO Global Geopark. Available online: http://www.unesco.org/new/en/natural-sciences/ environment/earth-sciences/unesco-global-geoparks/list-of-unesco-global-geoparks/greece/psiloritis/ (accessed on 16 January 2020).

47. Mingei Project. Keramion. Available online: http://www.mingei-project.eu/pottery-experience-keramion/ (accessed on 17 January 2020).

48. UNESCO. Mediterranean Diet. Available online: https://ich.unesco.org/doc/download.php?versionID=20926 (accessed on 16 January 2020).

49. UNESCO. Know-How of Cultivating Mastic on the Island of Chios. Available online: https://ich.unesco.org/ doc/src/26651-EN.doc (accessed on 17 January 2020).

50. UNESCO. UNESCO Moving forward the 2030 Agenda for Sustainable Development. United Nations Educational; Scientific and Cultural Organization Paris. Available online: https:/en.unesco.org/creativity/sites/creativity/ files/247785en.pdf (accessed on 12 February 2020).

51. Watkins, H.M.; Goodwin, G.P. Reflecting on sacrifices made by past generations increases a sense of obligation towards future generations. Personal. Soc. Psychol. Bull. 2019. [CrossRef] [PubMed]

(C) 2020 by the authors. Licensee MDPI, Basel, Switzerland. This article is an open access article distributed under the terms and conditions of the Creative Commons Attribution (CC BY) license (http://creativecommons.org/licenses/by/4.0/). 